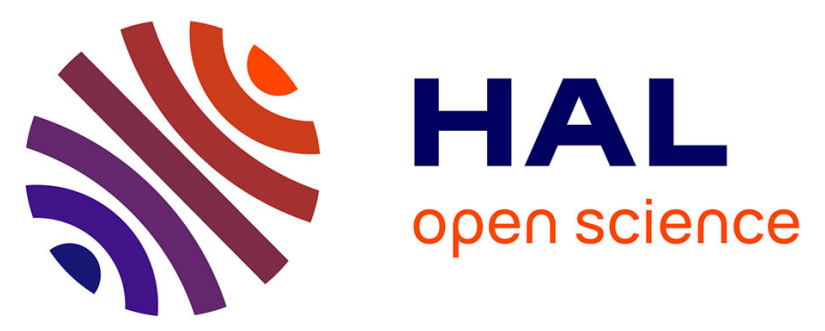

\title{
Pre-flight qualification test procedure for nanosatellites using sounding rockets
}

Leonardo Kessler Slongo, João Gabriel Reis, Daniel Gaiki, Pedro von Hohendorff Seger, Sara Vega Martínez, Bruno Vale Barbosa Eiterer, Tulio Gomes Pereira, Mario Baldini Neto, Matheus dos Santos Frata, Henrique Daniel Hamisch, et al.

\section{To cite this version:}

Leonardo Kessler Slongo, João Gabriel Reis, Daniel Gaiki, Pedro von Hohendorff Seger, Sara Vega Martínez, et al.. Pre-flight qualification test procedure for nanosatellites using sounding rockets. Acta Astronautica, 2019, 159, pp.564-577. 10.1016/j.actaastro.2019.01.035 . lirmm-02415535

\section{HAL Id: lirmm-02415535 \\ https://hal-lirmm.ccsd.cnrs.fr/lirmm-02415535}

Submitted on 15 Dec 2021

HAL is a multi-disciplinary open access archive for the deposit and dissemination of scientific research documents, whether they are published or not. The documents may come from teaching and research institutions in France or abroad, or from public or private research centers.
L'archive ouverte pluridisciplinaire $\mathbf{H A L}$, est destinée au dépôt et à la diffusion de documents scientifiques de niveau recherche, publiés ou non, émanant des établissements d'enseignement et de recherche français ou étrangers, des laboratoires publics ou privés. 


\section{Accepted Manuscript}

Pre-flight qualification test procedure for nanosatellites using sounding rockets

Leonardo Kessler Slongo, João Gabriel Reis, Daniel Gaiki, Pedro Von Hohendorff Seger, Sara Vega Martínez, Bruno Vale Barbosa Eiterer, Tulio Gomes Pereira, Mario Baldini Neto, Matheus dos Santos Frata, Henrique Daniel Hamisch, André Martins

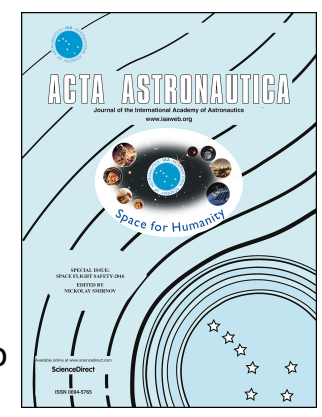
Pio de Mattos, Juan Pablo Florez Mera, Kleber Vieira de Paiva, Marcia Barbosa Henriques Mantelli, Luigi Dilillo, Eduardo Augusto Bezerra

PII: S0094-5765(18)31021-X

DOI: https://doi.org/10.1016/j.actaastro.2019.01.035

Reference: AA 7301

To appear in: Acta Astronautica

Received Date: 17 June 2018

Revised Date: 11 January 2019

Accepted Date: 27 January 2019

Please cite this article as: L.K. Slongo, Joã.Gabriel. Reis, D. Gaiki, P. Von Hohendorff Seger, S.V. Martínez, B.V. Barbosa Eiterer, T.G. Pereira, M.B. Neto, M.d.S. Frata, H.D. Hamisch, André.Martins.Pio. de Mattos, J.P. Florez Mera, K. Vieira de Paiva, M.B.H. Mantelli, L. Dilillo, E.A. Bezerra, Pre-flight qualification test procedure for nanosatellites using sounding rockets, Acta Astronautica (2019), doi: https://doi.org/10.1016/j.actaastro.2019.01.035.

This is a PDF file of an unedited manuscript that has been accepted for publication. As a service to our customers we are providing this early version of the manuscript. The manuscript will undergo copyediting, typesetting, and review of the resulting proof before it is published in its final form. Please note that during the production process errors may be discovered which could affect the content, and all legal disclaimers that apply to the journal pertain. 


\title{
Pre-flight qualification test procedure for nanosatellites using sounding rockets
}

Leonardo Kessler Slongo ${ }^{a, c, *}$, João Gabriel Reis ${ }^{\mathrm{a}}$, Daniel Gaiki ${ }^{\mathrm{b}}$, Pedro Von Hohendorff Seger ${ }^{\mathrm{e}}$, Sara Vega Martínez ${ }^{\mathrm{a}}$, Bruno Vale Barbosa Eiterer ${ }^{\mathrm{a}}$, Tulio Gomes Pereira $^{\mathrm{a}}$, Mario Baldini Neto ${ }^{\mathrm{a}}$, Matheus dos Santos Frata ${ }^{\mathrm{a}}$, Henrique Daniel Hamisch ${ }^{\mathrm{d}}$, André Martins Pio de Mattos ${ }^{\mathrm{a}}$, Juan Pablo Florez Mera ${ }^{\mathrm{e}}$, Kleber Vieira de Paiva ${ }^{\mathrm{f}}$, Marcia Barbosa Henriques Mantelli ${ }^{\mathrm{e}}$, Luigi Dilillog

Eduardo Augusto Bezerra ${ }^{a, g}$

\footnotetext{
${ }^{a}$ Department of Electrical and Electronic Engineering, UFSC, Florianópolis, Brazil

${ }^{b}$ Dipartimento di Elettronica e Telecomunicazioni, Politecnico di Torino, Italy

${ }^{c}$ SENAI Institute for Innovation in Embedded Systems, Florianópolis, Brazil

${ }^{d}$ Department of Automation and Systems, UFSC, Florianópolis, Brazil

${ }^{e}$ Department of Mechanical Engineering, UFSC, Florianópolis, Brazil

${ }^{f}$ Department of Mobility Engineering, UFSC, Joinville, Brazil ${ }^{g}$ CNRS/LIRMM, Université de Montpellier, France
}

\begin{abstract}
This work presents an innovative procedure to test nanosatellites subsystems on board sounding rockets. The procedure allows the subsystems to save their telemetry data during the whole rocket flight phases (including at the rocket lift-off). The subsystems of the FloripaSat (1U CubeSat) engineering model have been tested on board a VSB-30 rocket in order to validate their design and integration process. A dedicated embedded system has been proposed to operate as an electronic interface between the nanosatellite subsystems and the rocket electronics. Also, a system to process telemetry data was implemented on

\footnotetext{
* Corresponding author

Email addresses: slongo@eel.ufsc.br (Leonardo Kessler Slongo), jgreis@lisha.ufsc.br (João Gabriel Reis), daniel.gaiki@studenti.polito.it (Daniel Gaiki), pedro@labtucal.ufsc.br (Pedro Von Hohendorff Seger), sara.vega@posgrad.ufsc.br (Sara Vega Martínez), bruno.eiterer@grad.ufsc.br (Bruno Vale Barbosa Eiterer), tulio.gomes.pereira@grad.ufsc.br (Tulio Gomes Pereira), mario.baldini@posgrad.ufsc.br (Mario Baldini Neto), m.frata@grad.ufsc.br (Matheus dos Santos Frata), henrique.hamisch@grad.ufsc.br (Henrique Daniel Hamisch), andre.martins.mattos@grad.ufsc.br (André Martins Pio de Mattos), jpablo@labtucal.ufsc.br (Juan Pablo Florez Mera), kleber.paiva@ufsc.br (Kleber Vieira de Paiva), marcia.mantelli@ufsc.br (Marcia Barbosa Henriques Mantelli), Luigi.Dilillo@lirmm.fr (Luigi Dilillo), Eduardo.Bezerra@ufsc.br (Eduardo Augusto Bezerra)
} 
the ground station. The test procedure validates data frame definition, communication protocol specification, hardware integration specification, among other functionalities. Three FloripaSat subsystems have been tested: Electrical Power Subsystem (EPS); Telemetry, Tracking and Command (TT\&C); and On Board Data Handling (OBDH). Several nanosatellites' functions have been evaluated, including: battery monitoring; inertial measurement unit; temperature measurements; radio transceiver; and beacon. The flight results were considerably different from the results obtained at the laboratory. This has allowed important modifications to be made on the design of the CubeSat flight model as the revision on the radio circuit on TT\&C and the implementation of an external battery charger circuit for the EPS.

Keywords: Test procedure, design validation, nanosatellite, sounding rocket

\section{Introduction}

Nanosatellites have become one of the fastest ways to reach space due to their design simplicity and low-cost approach. They are small satellites (ranging from 1 to $10 \mathrm{~kg}$ ) with scientific and commercial purposes. This class of satellite has

5 emerged as an outstanding solution to allow students to have hands-on experience on actual space projects. The nanosatellites' launching "low price" and short development time attracts not only universities but also space enthusiasts. Many companies also have demonstrated interest to test their products and innovations in space through nanosatellites.

The CubeSat standard definition in 1999 (by Stanford and Cal Poly Universities) significantly increased the interest in small satellites. It defines a modular $10 \mathrm{~cm} \times 10 \mathrm{~cm} \times 11.35 \mathrm{~cm}(1 \mathrm{U})$ cubic shaped satellite, planned to operate in Low Earth Orbit and to be designed mostly with commercial off-the-shelf (COTS) components [1] 2].

However, along with the spread of the small sized satellite concept, a high failure rate has been noticed [3. Hardware and software design mistakes and failures on the integration process appear among some of the causes. Qualifi- 
cation and verification tests performed at laboratory are intended to avoid this problem [4. A Platform to guide hardware and software design for small satellites have also been proposed. Through component-based topology, the design platform allows software reutilization, diminishing design time and increasing the system reliability [5]. Also, a CubeSat mission design tool to estimate risk relationship appears as an interesting solution to predict and mitigate failures for small satellite missions [6]. Nevertheless, these strategies do not fully cover all the failure possibilities encountered during a real space mission.

Therefore, some strategies have been proposed to address this issue. Tests with atmospheric balloons [7] have emerged as a low cost and effective solution to test nanosatellites. Although it allows important preliminary communication tests between the satellite and the ground station, it does not evaluate the satellite behavior under the severe conditions of a rocket launching.

In a more elaborated solution, sounding rockets 8 , have been used for preliminary tests of nanosatellites, reducing failures before the mission. In this case, the sounding rocket ejects the nanosatellite after reaching a predetermined altitude. The satellite then communicates with a ground station for a period of time, before hitting the ground.

Although this is a more complete test than the ones performed with the atmospheric balloons, normally, nanosatellites only record/transmit data after they are released from the rocket. Actually, this occurs not only in preliminary tests with sounding rockets, but also during real missions. Normally, nanosatel-

40 lites are activated only several minutes after they are ejected from the rocket. This precludes nanosatellites to record/transmit data during the rocket flight. Many nanosatellite failures may occur during the rocket flight and may not be analyzed and understood due to the lack of information in this critical period of the mission. Several nanosatellites do not transmit after the rocket ejection 45 procedure and the developers have no idea about what may have caused the failure(s).

In this work an innovative procedure to test nanosatellites subsystems on board sounding rockets is presented. Instead of ejecting the nanosatellite, the 
proposed test is completely performed aboard the sounding rocket. This allows the nanosatellite to record data during all the phases of the rocket flight (including the launching). The nanosatellite starts operating before the rocket lift-off and it remains operating along the whole flight. The satellite data are transmitted to the rocket electronics, which retransmits the data to the ground station. With this strategy, one may have access to nanosatellite failures information that may occur during the rocket flight. Temperature increases during flight, high acceleration levels, cables issues due vibration, powering fails due to battery damage, are among some problems that may occur during launching and flight, which may be detected with the proposed test procedure.

An important remark here is that the proposed test procedure is not designed to substitute the others. It shall be interpreted as a complement to the aforementioned ones. The main technical contribution on this procedure is the capability to analyze satellite subsystems' behavior under the rocket launching and flight conditions. Although stratospheric balloons (or flying subsystems with drones) allow longer and more appropriate strategy for communication tests, they do not emulate the harsh environment of rocket launching and flight conditions. Even real nanosatellite missions can not provide data to this analysis, since the spacecrafts are placed in the P-POD (or equivalent launching device) during the flight, obligatorily in switch off condition. Laboratory qualification tests are essential, and they may be performed with the satellite subsystems collecting data. However, the physical phenomena are treated separately in each test (vibration, acceleration, mechanical shock, temperature cycling, etc.), and normally, in a lower level of intensity (specially for nanosatellite projects). These are the reasons why this test procedure has been proposed, believing that new data may arise from these suborbital flights, which will help nanosatellite designers in their projects.

Besides the procedures of nanosatellite's subsystem configuration and integration, this paper also describes the design of the Multi-Mission Platform (MMP), which is an embedded system conceived to allow nanosatellite tests (and other experiments) aboard sounding rockets. This platform is based on a 
modular architecture, which is a stack of three printed circuit boards (processing, acquisition and power units). Also, the ground station decoder software is presented in this work. It is based on LabVIEW language and its main application is to decode and save transmitted data along the rocket flight.

Finally, the paper presents the results of the FloripaSat (1U CubeSat) subsystems tested aboard a VSB-30 sounding rocket. Three engineering model subsystems have been tested: Electrical Power Subsystem (EPS); Telemetry, Tracking and Command (TT\&C); and On Board Data Handling (OBDH). The flight data of each subsystem are presented and discussed. This analysis will help preventing eventual failures on FloripaSat flight model, as well as, guide the design to improve some of its features.

The remaining of this paper is organized as follows: Section 2 presents the Multi-Mission Platform, designed to interface the nanosatellite subsystems and the rocket electronics; Section 3 explains how the microgravity experiment was planned, presenting the nanosatellite subsystems test configuration; Section 4 presents the ground station data processing software; Section 5 discusses the integration process; Section 6 explains the acceptance tests procedure; Section 7 discusses the suborbital flight results; finally, Section 8 concludes the paper.

\section{Multi-Mission Platform}

Sounding rockets have an electronic system (rocket electronics) responsible for receiving data from the payload experiments, and for transmitting them to a ground station. Therefore, any experiment which intends to transmit data to the Earth during the flight, shall communicate with the rocket electronics. The rocket electronics then sends the experiment data to a ground station through radio signal.

On the other hand, nanosatellites may communicate with ground stations by themselves, using their own radio and antenna. However, no external antennas (except the rocket main antenna) have been allowed for our sounding rocket flight. This has precluded the direct test of the TT\&C radio system. 
Consequently, the nanosatellite data had to be sent to the rocket electronics, Platform (MMP), shown in (Figure 2).

Besides the capability of testing nanosatellites, the Multi-Mission Platform is intended for scientific thermal experiments 11]10. The embedded system 


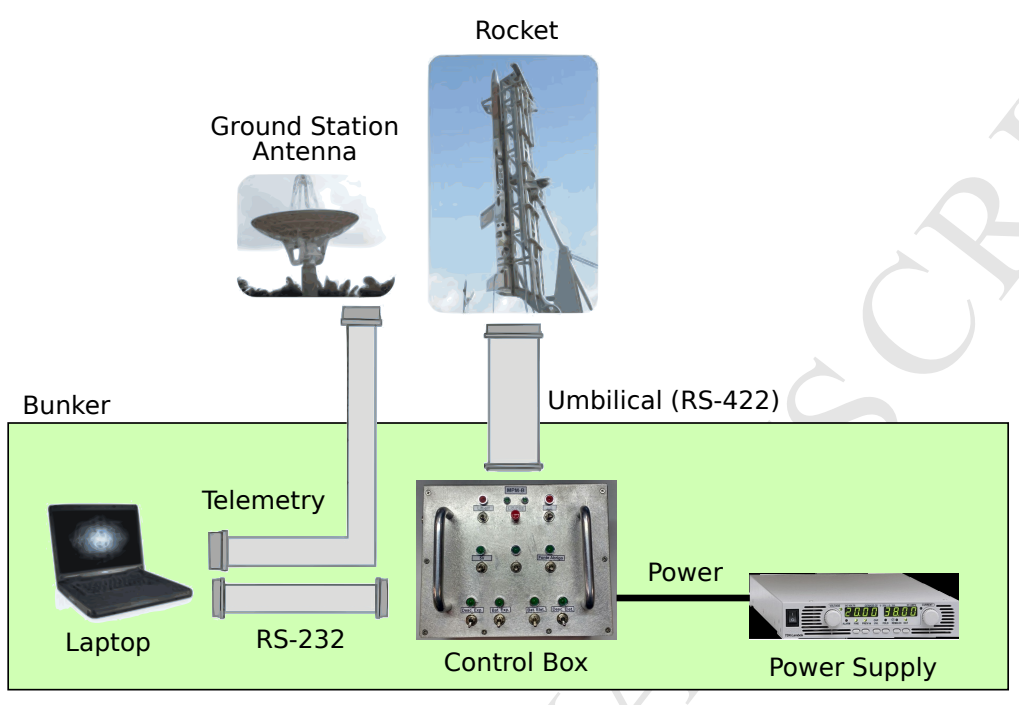

Figure 1: Rocket and bunker connections

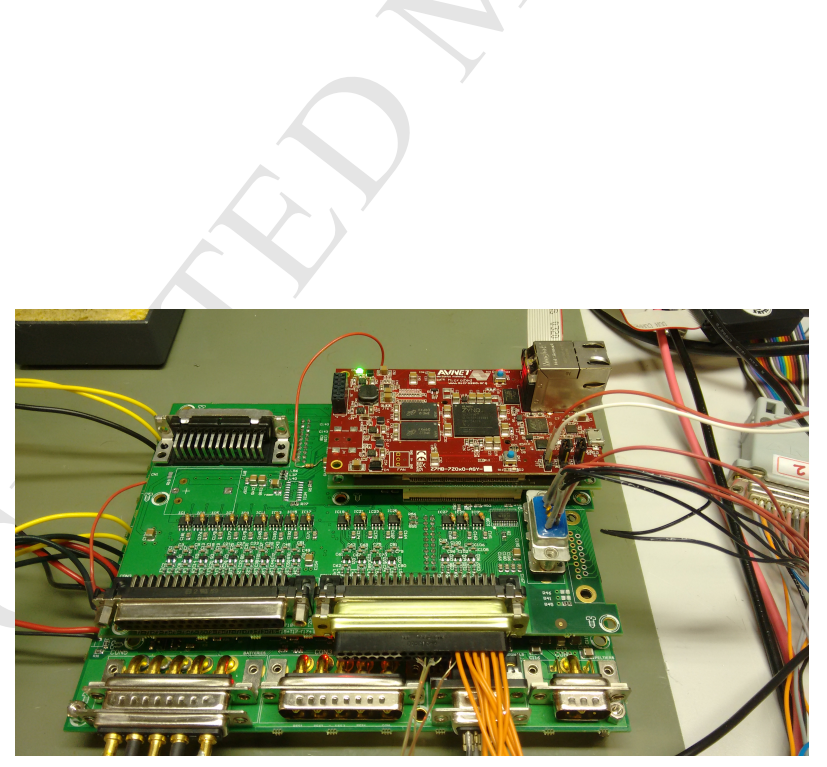

Figure 2: Multi-Mission Platform under test 
consists of a stack of three printed circuit boards: power; data acquisition; and processing. It is beyond the scope of this work to describe in details the Multi-Mission Platform. However, some characteristics are important to be mentioned, in order to comprehend the nanosatellite subsystems test procedure and the needed hardware/software resources to accomplish this goal.

According the mission requirements, the communication between the experiment electronics and the bunker shall be through RS-422 standard due to the distance between the bunker and the rocket launching platform (See Section 3.4 for detailed information on the communication protocol). Besides this, there shall be a second communication channel (between the experiment and the rocket electronics). For safety reasons both communication channels must be isolated, avoiding any eventual electrical instability to be propagated to the rocket electronics. This is the first reason to have a Multi-Mission Platform, since this kind of communication is not implemented in nanosatellites. The communication transceivers (ADM2682E) have been placed on the MMP data acquisition PCB. Figure 3 shows the transceivers electrical schematic, where signals with the label $u Z$ ed come/go to/from the MMP processing PCB.

Also in the mission requirements it is stated that the experiment electronics must present a safety mechanical turn on/off system. The power on command shall come from the bunker, using a control box. This is not a requirement for nanosatellites, but for the rocket mission. Therefore, the Multi-Mission Platform is provided with an isolated push button circuit to switch it on and off. Figure 4 shows the push button electrical schematic. Figure 5 shows the control box, placed at the bunker.

Finally, the rocket electronics keeps all the experiments informed regarding the lift-off and the microgravity condition. This is achieved by switching two pins/lines to ground (lift-off and $\mathrm{uG}$ pins). It is extremely important to the experiments to obtain these events triggering time reference in order to map the experiments results with the flight dynamics. Therefore, the Multi-Mission Platform has an analog circuitry to identify the events, as shown in Figure 6. Besides the analog circuitry, the embedded software is responsible for including 

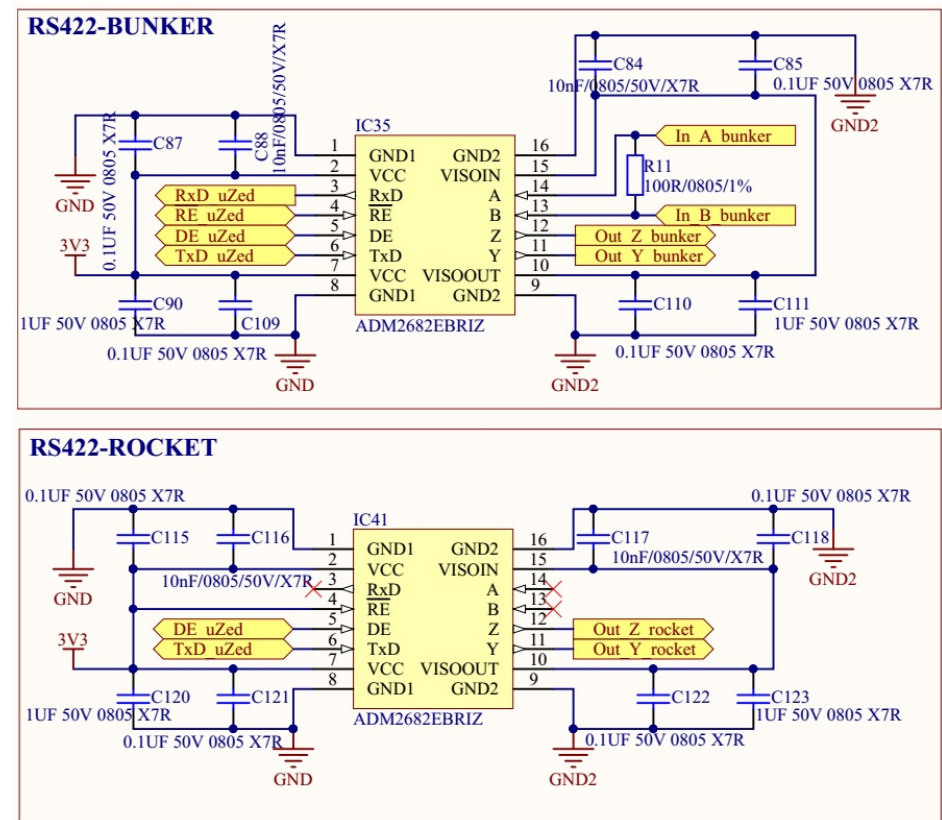

Figure 3: RS-422 transceivers electrical schematic

the events information on the data frame sent to the ground station (flight status byte).

\section{The Experiment}

The experiment to be performed in the sounding rocket is called New Medium Porous Technologies for Phase Changing Devices (MPM-A). It is carried out by an instrument which is implemented in a machined aluminum box with three compartments. The lower compartment contains both batteries (the thermal experiment batteries and the FloripaSat battery). The second compartment is entirely dedicated to the thermal experiment (Phase Changing Devices). The higher compartment contains the MMP and the FloripaSat subsystems. The experiment's goal is to test a thermal device, as well as the nanosatellite subsystems, using a Multi-Mission Platform as an interface for the devices under test and the rocket electronics. This section focuses on explaining the FloripaSat subsystems design and configuration procedures in order to test them aboard 


\section{Turn-on Push Button}

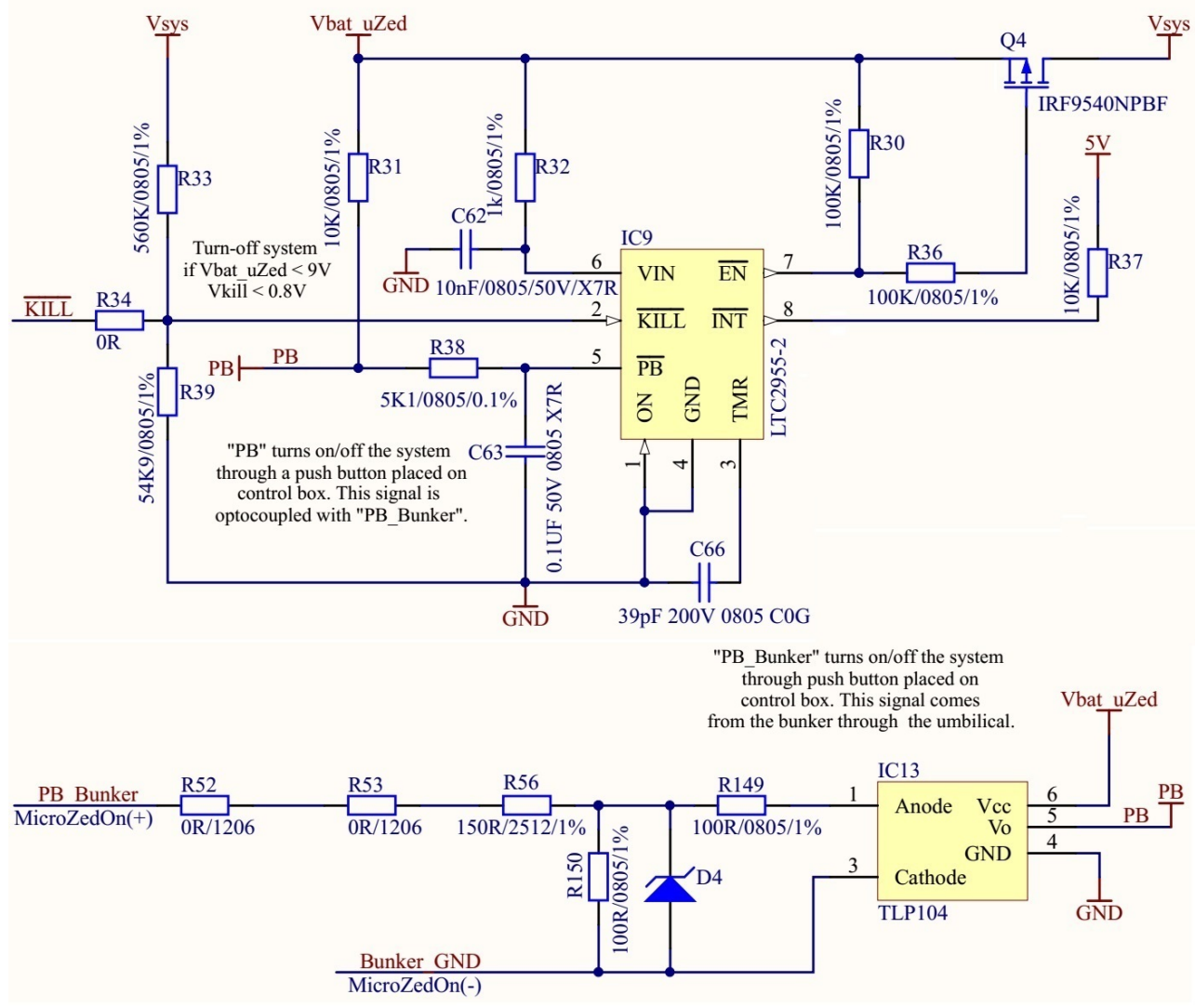

Figure 4: Push button turn on/off electrical schematic

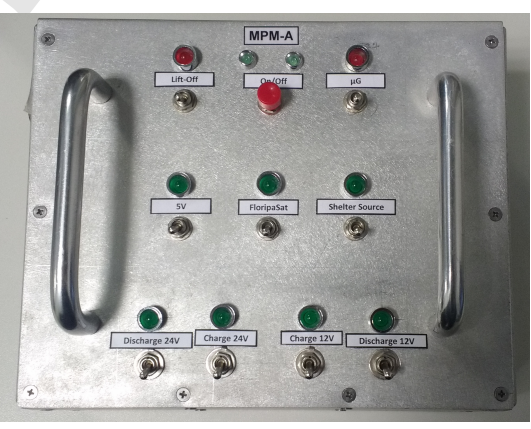

Figure 5: Control box 

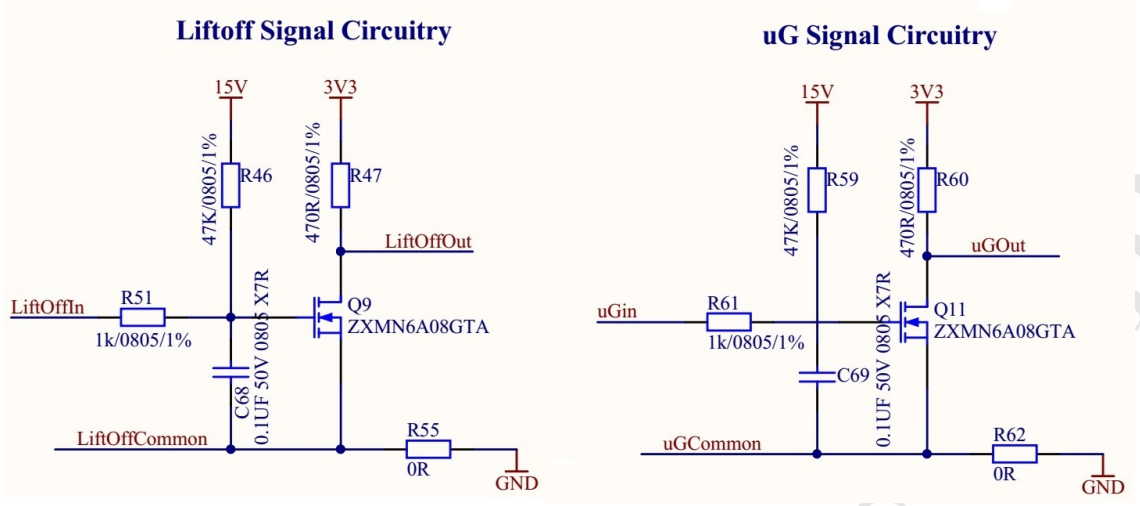

Figure 6: lift-off and uG circuitry

185 internal and external communication.

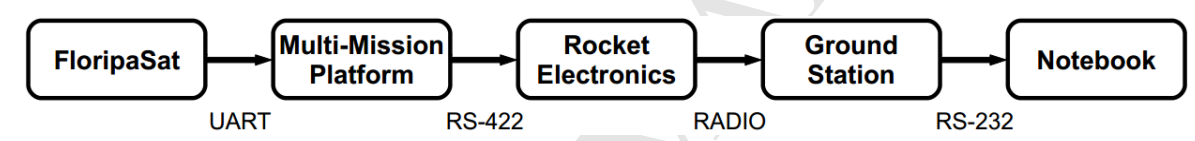

Figure 7: Experiment electronics communication diagram

\subsection{FloripaSat architecture}

FloripaSat main core has three subsystems: OBDH; TT\&C; and EPS. The OBDH and the TT\&C subsystems are located on the same PCB while the EPS is on a dedicated one. Combining functions of two different subsystems into a single subsystem/PCB is not a new idea and has been proven to be an effective approach for small satellites [12] 13. The flight model of FloripaSat shall have subsystems communicating with each other through different interfaces as shown in Figure 8 Normally, the OBDH shall build up the data frame and send it to the TT\&C subsystem (via SPI), which sends the data to the ground station (via radio transceiver). Also, in case the satellite receives a telecommand (via radio transceiver), the $\mathrm{TT} \& \mathrm{C}$ subsystem sends the data to $\mathrm{OBDH}$ (via $\mathrm{SPI}$ ) in order to process it. 
Figure 8 shows the FloripaSat configuration to operate in orbit, which differs

from the configuration of the sounding rocket experiment (Figure 91. As mentioned in Section 2, it was not allowed to place an external dedicated antenna to directly test the TT\&C communication. For this reason, a different communication configuration has been planned for the suborbital flight test with no hardware modifications made on the nanosatellite subsystems. For the sake of experiment electronics simplicity, only minor changes on the communication protocol were performed (see also Section 3.4 for complementary information.

In case of in-orbit configuration (Figure 8 the transceiver shall operate at $437.5 \mathrm{Mhz}$, transmitting and receiving signals, while the beacon shall only transmit (unidirectional TM downlink) at 145.9Mhz. On the other hand, for the sounding rocket experiment (Figure 9), the beacon radio was configured to transmit data internally to the transceiver radio. Even without the antennas, the radio frequency circuits should be able to send and to receive data to each other, due to their proximity (both circuits were placed at the same $\mathrm{PCB})$. To make the internal communication possible, the beacon radio transmitting frequency was changed to $437.5 \mathrm{MHz}$ (instead of keeping the original $145.9 \mathrm{MHz})$. Then, periodical messages were transmitted from the beacon radio to the transceiver, emulating what normally would be telecommands coming from the ground station to the transceiver.

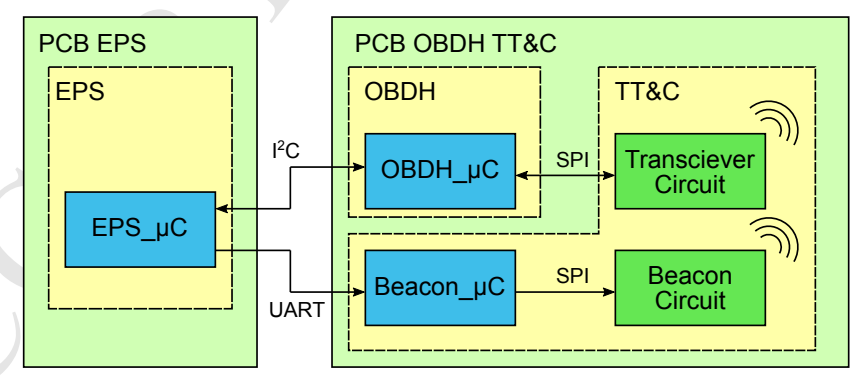

Figure 8: FloripaSat flight (in-orbit) model architecture diagram

The beacon microcontroller generates data (a simple 2 byte counter) and sends it through SPI to the beacon RF circuit. The data are transmitted to 
the transceiver circuit, which receives the RF signal, decodes it, and sends the data to the OBDH microcontroller via SPI. After receiving the beacon data, the $\mathrm{OBDH}$ requires the EPS data (via $\mathrm{I}^{2} \mathrm{C}$ main bus), concatenates the received data with its own measurements, and compose the complete FloripaSat data frame. The OBDH microcontroller sends the data frame to the Multi-Mission Platform Processing PCB via UART, and finally, the data frame is sent to the rocket electronics via RS-422. Figure 9 summarizes this data flow process.

An important remark is that the beacon configuration to change its transmitting frequency from $145.9 \mathrm{Mhz}$ to $437.5 \mathrm{MHz}$ is performed externally, through the computer, writing in the chip internal memory. This means that no software is changed on the beacon side when comparing the in-orbit configuration scenario and the sounding rocket experiment.

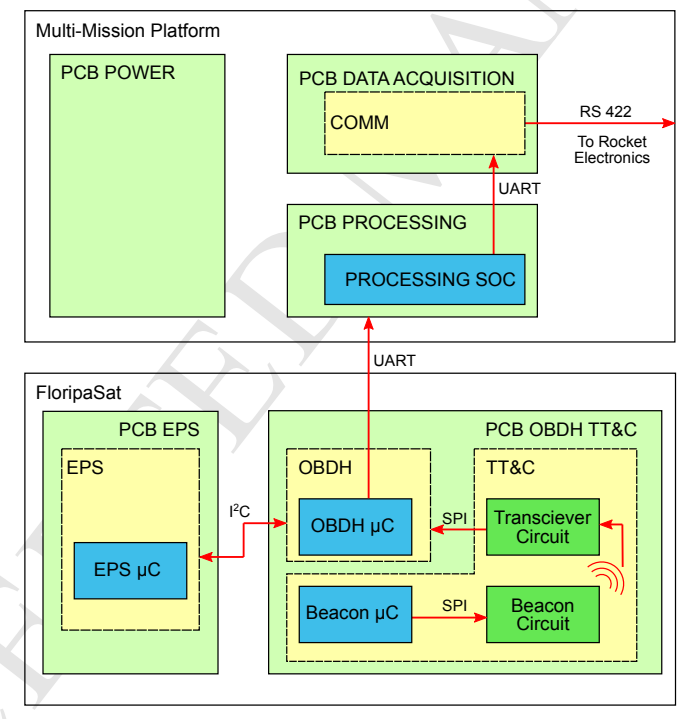

Figure 9: FloripaSat engineering model architecture diagram for the suborbital test

\subsection{Mission frame definition}

Before discussing in details the FloripaSat's telemetry data, it is important to recall that the Multi-Mission Platform acquires data not only from the FloripaSat subsystems, but also from a thermal experiment, as previously stated. Be- 
fore sending the data to the ground station, the Multi-Mission Platform packs the thermal experiment data (174 bytes) and the FloripaSat data (42 bytes) into a multiple frames containing 432 bytes (216 payload bytes) every sampling period. The Multi-Mission Platform transmits the acquired data split into 72 frames of 6 bytes. The first 58 frames concern the thermal experiment data frame and the last 14 frames concern the FloripaSat telemetry data. Further discussions regarding each of the thermal experiment data is beyond the scope of this work. Therefore, the focus is kept on the FloripaSat. Figure 10 shows the structure of the data frame transmitted by the Multi-Mission Platform to the ground station.

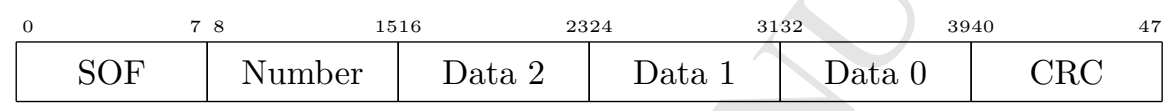

Figure 10: Data frame structure with the corresponding bit number above each field.

The Start of Frame (SOF) is the first byte transmitted to indicate to the ground station the beginning of a new frame and it is followed by the frame number used to identify the data field transmitted. The 3 following data bytes are the payload itself. The MMP generates a Cyclic Redundancy Check (CRC) considering 4 bytes of each frame: Number and the 3 data bytes. Due to the unreliable link between the ground station and the sounding rocket, the frame has only 3 payload bytes. By doing so, we avoid discarding a larger payload in case of data corruption at the expense of the overhead in each frame. An analysis on the data loss due to communication failures during the rocket flight is presented in Section 7 .

\subsection{FloripaSat telemetry data}

Unlike the thermal experiment data, the FloripaSat telemetry data are not generated at the Multi-Mission Platform. It is the nanosatellite's OBDH task to acquire data from subsystems and sensors, to generate an internal frame and to send it to the MMP through UART. Notice that the FloripaSat frame is then split by the MMP and encapsulated in its own frame previously described. 
Since the FloripaSat OBDH main function is to control the nanosatellite tasks execution and build up the transmitted data frame, only few bytes were

nication integrity verification). Later, in a second verification level, the ground station processing software verifies the mission main data frame (rocket elec- 
tronics - ground station radio communication integrity verification - see Section

295 4).

Therefore, in order to the MMP to identify the FloripaSat data frame (higher level verification), three bytes were defined as Start of Frame, and three bytes as the End of Frame (EOF). These bytes allow the ground station decoder software to correctly identify the beginning of new upcoming data frames, after receiving an eventual broken frame. Finally, an eight bit CRC has been implemented to identify corrupted data frames. The list containing all field in FloripaSat data frame is shown next.

1. Start of Frame (SOF), 3 bytes: Start of Frame delimiter.

2. OBDH timestamp (s), 2 bytes: seconds representation of OBDH counter.

3. OBDH timestamp (ms), 2 bytes: milliseconds representation of OBDH counter.

4. OBDH temperature, 2 bytes: OBDH internal temperature.

5. OBDH status, 1 byte: OBDH internal status.

6. IMU acceleration $\mathrm{x}$-axis, 2 byte: IMU acceleration in the $\mathrm{x}$-axis.

310

7. IMU acceleration y-axis, 2 byte: IMU acceleration in the y-axis.

8. IMU acceleration z-axis, 2 byte: IMU acceleration in the z-axis.

9. IMU angular rate $\mathrm{x}$-axis, 2 byte: IMU angular rate in the $\mathrm{x}$-axis.

10. IMU angular rate $y$-axis, 2 byte: IMU angular rate in the y-axis.

11. IMU angular rate z-axis, 2 byte: IMU angular rate in the z-axis.

315

12. Radio counter 1,2 byte: TT\&C most significant transmitted data counter.

13. Radio counter 2,2 byte: TT\&C less significant transmitted data counter.

14. Battery current 2, 2 byte: Current drawn from battery.

15. Battery voltage 1, 2 byte: Voltage on battery 1 .

16. Battery voltage 2, 2 byte: Voltage on battery 2 .

320
17. EPS temperature, 2 byte: Internal EPS temperature.

18. Electric charge, 2 byte: Battery remaining electric charge.

19. EPS status, 1 byte: Status register for the battery protection circuit.

20. CRC, 1 byte: CRC of all previous field except for SOF.

21. End Of Frame (EOF), 3 bytes: End of frame delimiter. 


\subsection{Communication protocol specification}

The VSB-30 electronics receives data from the payload through an RS-422 interface. This interface is not common on nanosatellite applications due to its voltage requirements. Instead of including an RS-422 driver in the FloripaSat, in order to attend the rocket requirements, it was placed on the Multi-Mission Platform (see Section 2).

Two different communication channels have been implemented on the MultiMission Platform (see Figure 3). The first one is dedicated to the communication between the bunker and the experiment electronics, before the rocket lift-off, where the main limitation on this channel is the distance between the bunker and the rocket (which is below the RS-422 standard limitation of $1500 \mathrm{~m}$ ). This is a point to point channel and could be taken to the physical bandwidth limits defined by the standard. The second channel concerns the communication of the experiment and the rocket electronics used to transmit the data to the ground station. It is limited in bandwidth due to all the payload experiments on board the rocket using the same channel. The maximum allowed baud rate for each experiment on board was 57,600 bps.

The Multi-Mission Platform receives FloripaSat data frames through a UART port. There is no synchronization protocol between them but, to ensure that no data are lost due to overflows, the Multi-Mission Platform defines a maximum UART baud rate and guarantees that below that limit, it is able to store and transmit all the received data frames. The Multi-Mission Platform initially parses the received data to ensure it follows the data frame specification presented previously. It initially searches for the SOF bytes and then it buffers all the data it receives, until it finds the EOF bytes. Next, it checks if the CRC is valid. If it is, the data frame is stored for transmission with the rest of the experiment data. In case of a mismatch in the $\mathrm{CRC}$, the data frame is discarded.

One important remark is that the communication between the EPS and

the beacon microcontroller, which shall occur for in-orbit configuration (see Figure 8 has not been tested on the sounding rocket test procedure (see Figure, where there is no EPS-beacon UART communication). The reason for this 
decision is that, in a real mission, the EPS data goes to the ground station via the beacon radio, and not back to the $\mathrm{OBDH}$, as designed for this experiment. Implementing this for the sounding rocket mission would allow testing the EPS - beacon communication, but on the other hand, it would cause an overhead on the OBDH functionality. The OBDH would have enough computation resource for this task, but the embedded code would be considerably different from the OBDH flight software for the satellite mission in orbit. Therefore, the decision was to keep it simple, not testing this portion of the protocol in the suborbital flight.

A protocol modification was also necessary on the beacon/transceiver communication. In case of in-orbit operation, when a telecommand is received by the transceiver, the OBDH decodes it and executes the command. In the suborbital mission configuration, the "telecommand" received by the transceiver (sent by the beacon) does not causes the OBDH to execute any task. In fact, this "telecommand" is a counter running on the beacon microcontroller, which has its current value sent periodically (every each increment) to the transceiver. This was made to simplify the internal communication test, since the beacon is not supposed to generate telecommands. This internal communication between beacon/transceiver shall never occur in orbit, and for this reason the proposed 375 protocol has been simplified, with the intention to test the RF link only.

\section{On-ground data processing software}

In order to send as much information as possible to the ground station, both the thermal experiment and the FloripaSat data were transmitted in raw format. As explained in Section 3.2 , the data were packaged to frames with SOF, Number and CRC bytes. Therefore, a software application was implemented to allow data unpacking, processing and logging at the bunker during the rocket flight. The software was based on LabVIEW language, running in a notebook placed at the bunker.

As mentioned previously, the mission main data frame has 432 bytes (72 
frames of 6 bytes) where the last 84 (14 frames of 6 bytes) are the ones related to the FloripaSat. Therefore, the LabVIEW software has been designed to identify the FloripaSat frames in order to unpack and process them. Since each frame contains 3 bytes of data (the other 3 bytes are SOF, Number and CRC), there are 42 data bytes in total which contain information from the FloripaSat (payload bytes). These are the bytes that must be converted by the LabVIEW software into readable information to be saved during the rocket flight.

Figure 11 shows the FloripaSat data frame extraction from the mission data frame. It occurs in two parallel paths. The first one (upper part of Figure 11, extracts the frames 60 to 71, which are the FloripaSat data frames excepting the EOF and the SOF. These 12 frames go to the Fsat subVI in order to be processed. This VI converts the data to readable values, sends them to the LabVIEW Front Panel (left hand column (Data) in Figure 12. For each data value on the LabVIEW Front Panel (left hand column), there is a label to identify it (e.g. Clock(s), Clock(ms), Internal Temp., etc.). After the data unpacking and conversion, they are saved in a .csv file.

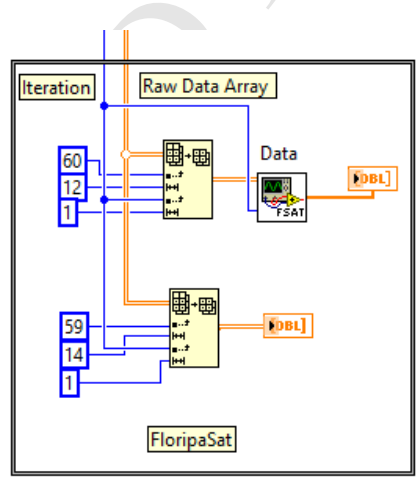

Figure 11: LabVIEW block diagram of FloripaSat data unpacking process

The second path in Figure 11,is dedicated to extract frames 59 to 72 from the main frame, which contains all the FloripaSat data (including EOF and SOF). This vector is directly sent to the LabVIEW Front Panel (right hand column (Raw Data) in Figure 12. Every line of this column shows a 3 byte payload, 

side of Figure 12. After this extraction, the raw data are saved in a .csv file, for redundancy and posterior processing, in case it is necessary.

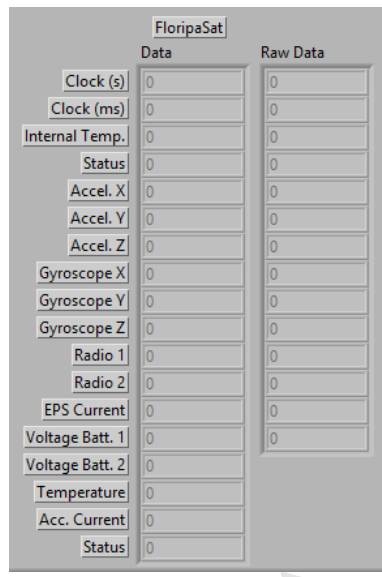

Figure 12: LabVIEW front panel for the FloripaSat received data

Figure 13 shows the Fsat subVI. This block receives the mission frames 60 to 71 (72 bytes - 12 frames of 6 bytes) and extracts only the FloripaSat 36 payload bytes (12 frames with 3 payload bytes each). This process removes the frame bytes SOF, Number and CRC. This is achieved through a for loop, which does 12 iterations: each iteration receives a frame, extracts only the 3 payload bytes from it and allocates them to a new vector. At the end of the 12 iterations, the 36 payload bytes are stored in a vector that is forwarded to the next processing 415 block.

The second processing block receives the vector with 36 bytes and, through another for loop, rearranges them in a vector with 18 positions. This reorganization is performed in order to generate the 18 data observed in Figure 12 . timestamp, accelerometer, gyroscope, current, voltage, etc. In addition to rearranging the data, some of them go through formulas in order to be converted to meaningful data. This 18 position vector is the output of the Fsat subVI. It is reinserted into the mission data array (which also contains the processed data from thermal experiment) and then saved to a file. Therefore, the on-ground 
data processing software allows the converted and raw data being visualized during the flight and saved in files for further analyses.

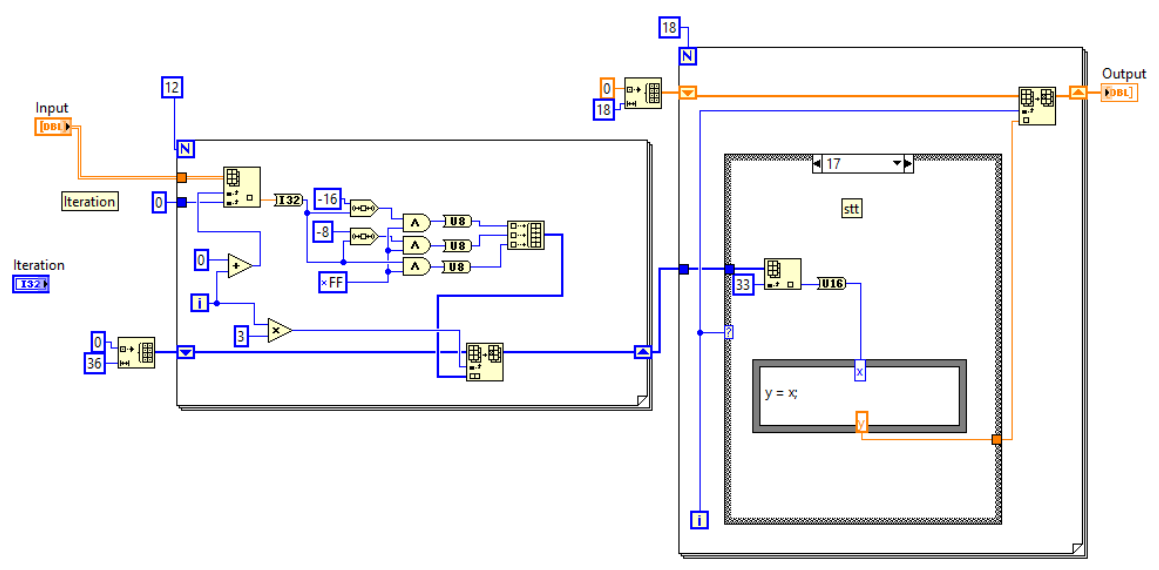

Figure 13: LabVIEW subVI of FloripaSat data decoder

\section{Integration process}

The nanosatellite subsystems integration to the MPM-A instrument has occurred in three levels. First, the FloripaSat subsystems have been integrated one with each other. Then, the subsystems were integrated with the MMP. Finally, the FloripaSat subsystems and the battery were integrated in the MPM-A machined aluminum box. The first integration level started with the nanosatellites subsystems placed side-by-side, connected by wires, with their software running in debug mode. Initially, powering tests have been conducted, using voltages supplies instead of the battery, in order to certify that the EPS was correctly suppling power to the OBDH and TT\&C subsystems. After passing the preliminary powering tests, the FloripaSat battery have been assembled. It consists of two lithium ion battery cells connected in series (ICR18650-30B) and covered in silicon. Three wires were routed from the battery (lower instrument box compartment) to the subsystems (higher instrument box compartment). The wires were connected to the battery GND, to the intermediate voltage point (between 
two battery cells), and to the total voltage point.

After that, the FloripaSat PCBs internal communication were tested. To achieve this, a simple periodic routine was implemented to send a vector of fixed bytes from EPS to OBDH through $\mathrm{I}^{2} \mathrm{C}$ protocol. To verify the correctness on this internal communication, the OBDH was configured to send the vector of bytes to a computer, through UART. After validating the internal communication, the EPS software has been modified to acquire real data from its sensors. Also, the OBDH included real sensors data to the FloripaSat data frame sent to the computer. Finally, the communication between TT\&C and OBDH has been validated. A counter has been implemented on the beacon microcontroller, for each performed transmission to the transceiver. The SPI communication between these two subsystems has been validated by checking the counter increases on the final data vector sent from the OBDH to the computer.

Then, the integration with the MPP initiated. Both electronics were placed side-by-side and the activating system has been wired up from MPP to FloripaSat subsystems. It is important to understand how this solution has been implemented. Nanosatellites are normally turned on by deployment switches attached to the satellite's structure [14. These switches remain deactivated when the satellite is inside the launcher, causing the satellite to electrically disconnect the power system from the others. Once the satellite is launched, these switches electrically close and the satellite is powered on.

The activating circuit schematic, considering the case of a satellite mission, is shown in Figure14. Two P channel MOSFETs are connected to the deployment switches and to the Remove Before Flight (RBF) to power off the subsystems. When the RBF is placed or/and the deployment switches are open (this is the condition when the satellite is placed in the deployment mechanism, e.g. P-POD), the P-channel MOSFETs do not allow the current to flow from the battery to the load. When the satellite is placed in the deployment mechanism, the RBF shall be removed, since the deployment switches will remain opened. When the satellite is released in orbit, theses switches close, and the satellite is powered. 
However, in the sounding rocket mission, this activating system needed to be changed. First of all, there was no deployment mechanism to place the subsystems in, since they were not deployed from the rocket, as in a common satellite mission. Secondly, the FloripaSat subsystems shall be activated by the MMP, when it receives the lift-off signal. Therefore, the FloripaSat EPS hardware was configured in order to attend the sounding rocket mission requirements.

It is important to differentiate hardware configuration from modifications in hardware. The first concept is related to components values that may be changed, or components that may or not be assembled in the PCB. On the other hand, the hardware modification concept implies on changes on the PCB circuit, which results in a new PCB design or in irreversible re-manufacturing changes on the PCB (e.g. cutting tracks). From the original FloripaSat switching circuit (Figure 14 to the modified version (Figure 15, only a new hardware configuration was defined for the EPS. A FloripaSat engineering model pair of PBCs was assembled specifically for the sounding rocket campaign, therefore the hardware could be previously configured.

The resistor R26 in Figure 15 was mounted with $133 k \Omega$, instead of $0 \Omega$ (as in the original schematics shown in Figure 14. The Remove Before Flight connector was not mounted on the PCB, and its terminals have been shortcircuited. End-rail switches connectors have not been mounted (since those switches are not used in the sounding rocket experiment); jumpers were mounted in their places. Resistor R80 has not been mounted (Figure 14 and the NPN transistor has been mounted in its place, with the transistor's base pin being wired to the Multi-Mission Platform (Enable Multi-Mission Platform). As the P-channel MOSFET only conducts when it has a low level voltage in its gate, this configuration allowed the Multi-Mission Platform to enable the FloripaSat subsystems, as shown in Figure 15 .

After that, the FloripaSat was connected with the Multi-Mission Platform to test the switch on procedure and communication through UART. The data generated by FloripaSat were sent to the Multi-Mission Platform and then sent to a computer running the LabVIEW data processing software, which allowed 


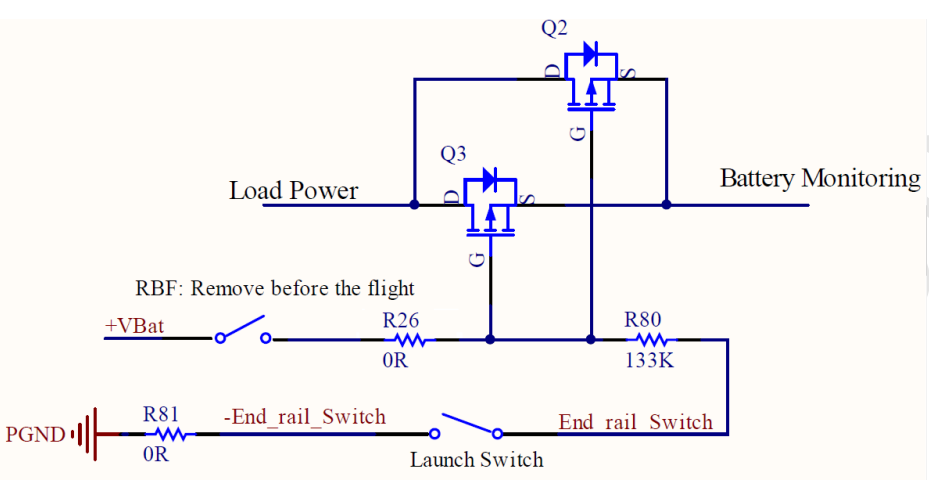

Figure 14: Original switching on circuit from FloripaSat

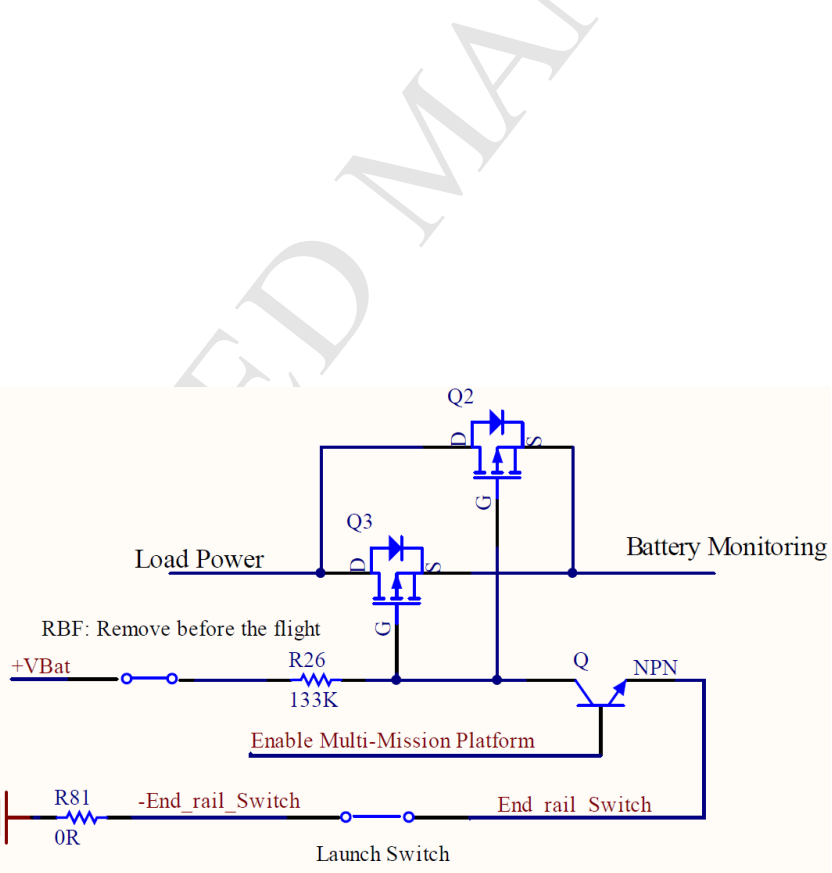

Figure 15: Modified switching on circuit to turn on FloripaSat subsystems by MMP 
the debugging of the FloripaSat running the release software version. During this phase of the integration, software bugs were found in the LabVIEW software which led to additional test procedures, such as the usage of an oscilloscope to verify the frequency which data were being sent by the OBDH.

Then, the flight version of the software was programmed into the boards before stacking them, since the programming connectors were not accessible after mechanical integration. After this step, the FloripaSat PCBs were stacked. The electrical connection between boards is performed through a PC-104 style connector. Metallic spacers have been screwed between PCBs to guarantee mechanical stability. The connections between FloripaSat subsystems and the MMP were made by soldering wires. To increase the mechanical stability of electronic components on the PCBs, silicone was poured over the most sensitive areas of all subsystems. Figure 16 shows a photo of the FloripaSat OBDH, EPS and TT\&C subsystems under integration.

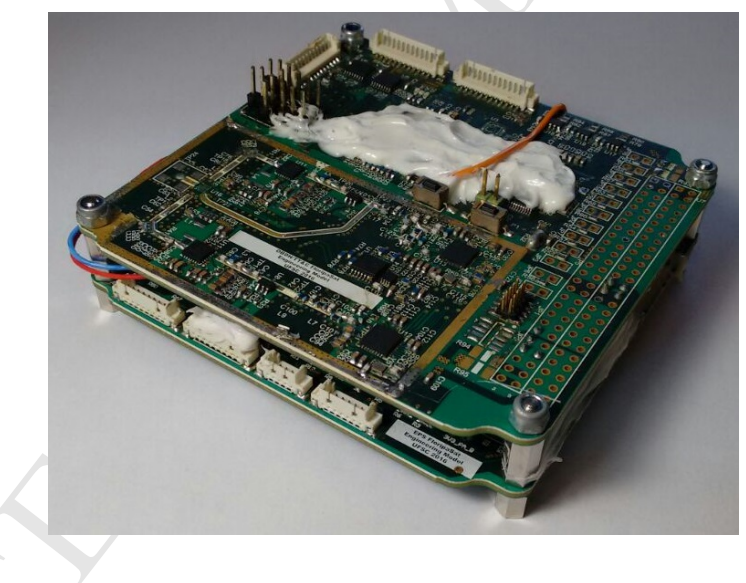

Figure 16: FloripaSat OBDH, EPS and TT\&C subsystems under integration

After this test procedures, the FloripaSat subsystems were attached to the top part of the MPM-A aluminum case. Also, the batteries have been placed at the lower compartment of the aluminum case. Figure 17 shows a photo of the MPM-A instrument under integration, just before closing it. 


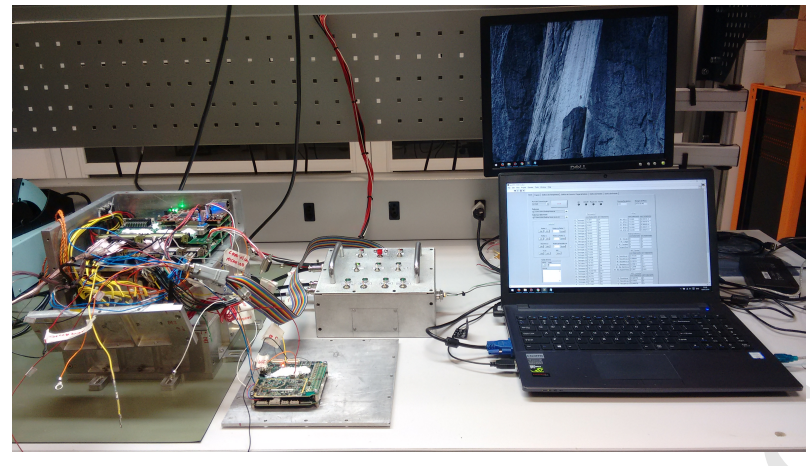

Figure 17: FloripaSat subsystems under integration with the MPM-A instrument

\section{Acceptance tests}

After the integration process, carried out at the Federal University of Santa Catarina, the MPM-A has been sent to the Department of Aerospace Science and Technology (DCTA, in Portuguese), from the Brazilian Air Force, to be submitted to the flight acceptance tests. This test procedure differs from the flight qualification tests, where the experiments are submitted to similar conditions to the rocket flight. On the vibration test, the instrument (the complete aluminum casing with the MPP, FloripaSat subsystems, thermal experiments and batteries) has been submitted to frequencies from $20 \mathrm{~Hz}$ to $2 \mathrm{kHz}$ in aleatory mode. The tests were performed for the three axes (x, y and $\mathrm{z}$ ), separately. The acceptance test level for the payload instruments, adopted for this sounding rocket mission was $6.1 \mathrm{gRMS}$. In case of qualification tests, it would be 13.2 gRMS.

The reason for applying acceptance tests, which are less severe than qualification tests, is that such research experiments normally do not have a spare sets for severe tests that may cause irreversible damages to them. The lacking of spare sets are normally caused by the experiments' complexity, high cost and long development time. The same situation applies to nanosatellites. The intrinsic idea of low cost and fast development time, comparing to bigger satellites, precludes the research groups of fabricating two identical versions of their 
spacecrafts. Therefore, the nanosatellites flight model are normally not submitted to severe qualification tests. This may also be one of the causes for a high failure rate on this class of satellites.

Therefore, this work suggests firstly submitting the nanosatellite subsystems engineering model to an acceptance test, which is faster, cheaper and less degrading. Then, testing these subsystems under flight, with the subsystems operating along the whole flight, recording data on severe and real flight conditions. Engineering model subsystems are far cheaper than the whole integrated flight model nanosatellite, which includes mechanical structure, solar panels, antenna, etc. In case the subsystems fail or crash at any moment on the flight, the data are recorded and may be further analyzed, avoiding committing the same design mistakes on the flight model.

After this level of tests with the engineering model subsystems, the flight model may be more reliably designed, and then the acceptance tests will be enough to preclude the satellite failures on the real mission. Besides this, having an in-flight test during the satellite design phase motivates the team and sets up hard deadlines which are important for students to accomplish. Figure 18 shows a flow chart summarizing the proposed test procedure. Each box represents a step on the procedure, which were described in more details through the paper's sections.

Figure 19 shows the MPM-A instrument right before the acceptance tests at the DCTA. Due to military restrictions, it was not possible to take pictures of the MPM-A instrument under acceptance tests. The performed tests were: mass test; fit test; and vibration test. Vacuum tests were not needed because the rocket payload modules were pressurized and hermetically sealed.

After the MPM-A instrument has passed the acceptance tests, the rocket payload and its electronics were integrated and submitted again to a new acceptance test. Next, when the rocket payload has passed through all the acceptance tests, it was partially dissembled to be sent to the Alcântara Launching 570 Center. After the final integration at the launching center, the rocket was ready for flight. 

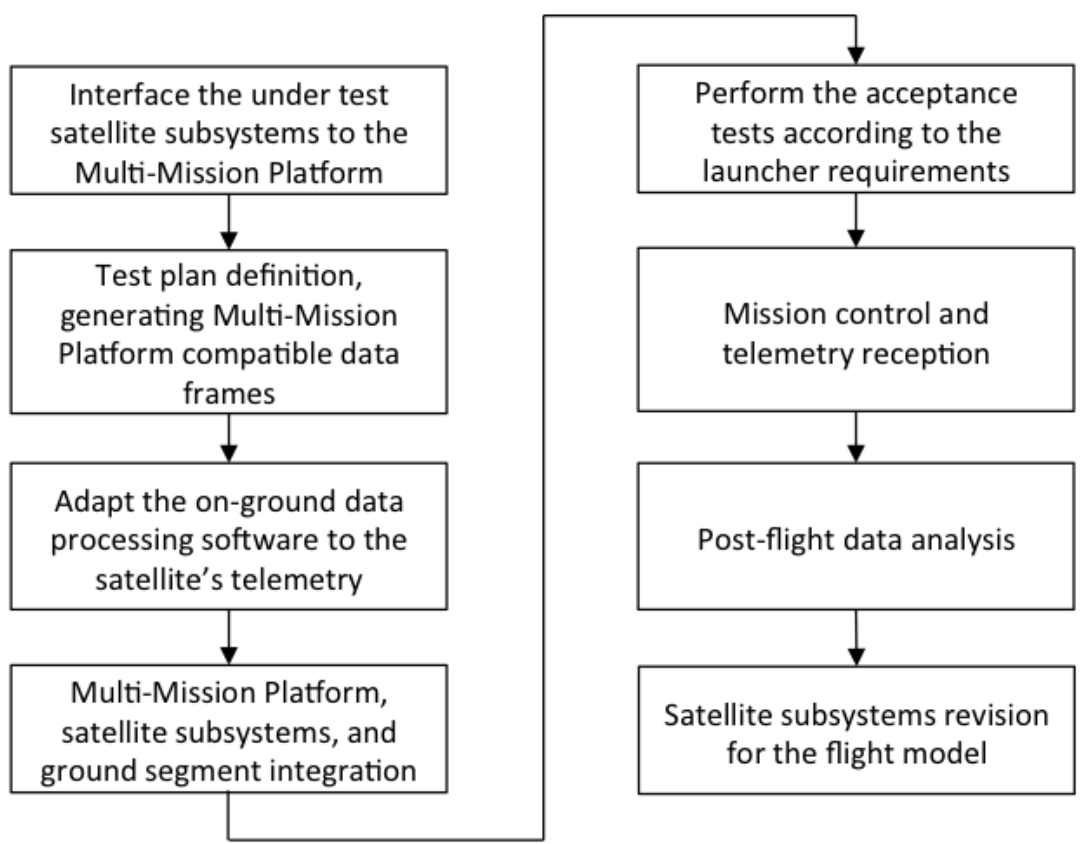

Figure 18: Proposed test procedure

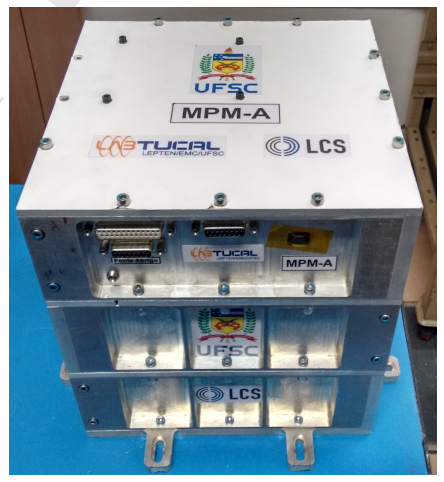

Figure 19: MPM-A instrument after acceptance tests - ready for flight 


\section{Flight results}

This section is dedicate to present the nanosatellite results during the sounding rocket mission. The intention is to verify the nanosatellite operation under severe conditions, once the nanosatellite was operating even before the rocket lift-off.

However, before analyzing the flight data, it is important to briefly describe the mission phases, in order to correctly interpret the results.

- Phase 1 - The MMP and the FloripaSat have been turned on with the rocket at the launching platform 30 minutes before the rocket lift-off. At this moment, there was a physical communication between the MMP and the bunker, through the umbilical cable. This allowed receiving data from the FloripaSat and also charging its battery (see Figure 1 ).

- Phase 2 - Three minutes before the launching, the batteries charging process (for both the MPP and the FloripaSat) was intentionally interrupted, due to safety reasons, as a mission requirement. However, the MMP and the FloripaSat remained operating and sending data to the bunker through the umbilical cable.

- Phase 3 - At the lift-off moment, the umbilical cable was disconnected and the rocket entered on flight mode. From this moment on, data have been sent through the rocket radio communication system only. Flight data were received by the ground station antenna and sent to the bunker to be processed by the on-ground data processing software.

- Phase 4 - After nearly 1 minute the rocket should have entered the microgravity phase. Unfortunately, due to the rocket malfunctioning, which is not related to the experiments, this phase was not observed in this flight, and the respective related data are not available.

- Phase 5 - After less than 3 minutes the rocket payload parachute system has been activated and the payload safely landed on sea nearly 6 minutes after the lift-off. 
The rocket electronics generates two important signals to the payload experiments: lift-off and microgravity signals. These signals are important time references for data analyses. However, since there was no microgravity condition in this flight, only the lift-off signal has been received on the MMP. In our experiment, besides the lift-off signal time reference, the batteries charging interruption (which has occurred before the lift-off) has also been registered in order to be considered on mission data analyses. The parachute activating system generates no signal to the experiments, therefore, there is no specific time reference for this flight event.

The first results to be analyzed are the FloripaSat EPS data, acquired before and during the flight by the battery monitoring chip. This chip communicates with the EPS microcontroller and measures battery cells voltages, battery current, battery remaining electric charge and temperature. The acquired data are sent from the EPS microcontroller to the OBDH microcontroller through the $\mathrm{I}^{2} \mathrm{C}$ protocol, as shown in Figure 9.

Figure 20 shows the FloripaSat battery cells voltages behavior along the time. During the phase that FloripaSat was operating with the rocket at the launching platform (nearly 27 minutes), one may note that both battery cells remained nearly at the same voltage level. When the charging process was interrupted, the cells voltage instantaneously dropped, but remained on the expected operating level. Also, it is possible to identify a slightly voltage drop for both battery cells during the flight, due to the discharging process. No unexpected behavior has been identified on this result.

Figure 21 shows the FloripaSat instantaneous battery current behavior during the mission. Negative currents represent the battery discharging behavior. This plot shows that, even during the charging period, the battery current remained negative. The first reason for this behavior is that the subsystems were operating during the battery charging process and they demanded more current than the charging current. The second reason was the adopted charging method-

${ }_{630}$ ology, which is constant voltage charging. It is well known from the literature that constant voltage is not the most appropriate way to charge lithium-ion 


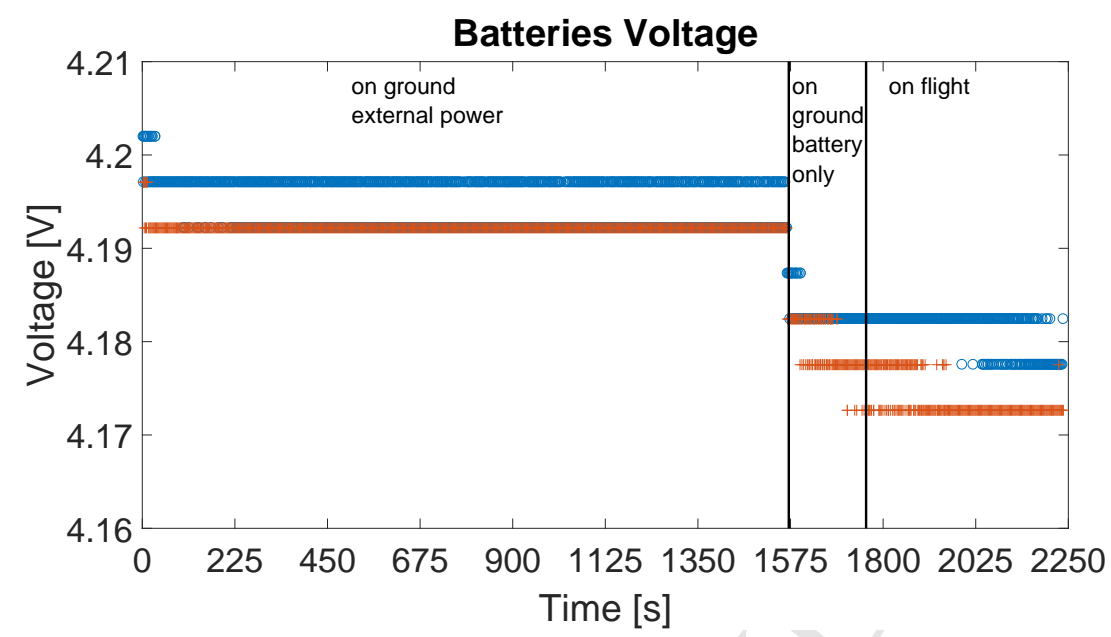

Figure 20: Battery cells voltages

batteries [15], however, this was the safest manner to charge the battery at that critical moment. Constant current charging could lead the battery cells to over-voltage state (there was no external charging control system available), which would activate the battery protection system minutes before the flight. In order to avoid this unnecessary risky condition, a most conservative approach has been chosen.

This charging issue faced during the mission has led to a charging circuit design, which will be used for battery external charging procedure before the

FloripaSat flight. Also, the research group is testing a constant current charging circuit block to be implemented between the solar panels and the battery. This could reduce the EPS harvesting energy capability, but extend the battery lifetime. Therefore, this different architecture is under consideration for future EPS designs.

Figure 21 also shows that, after the lift-off, the battery current remained constant along the whole flight, on the expected level of nearly $23 \mathrm{~mA}$ (global current consumption of all modules).

Figure 22 shows the electric charge of the battery before and during the 


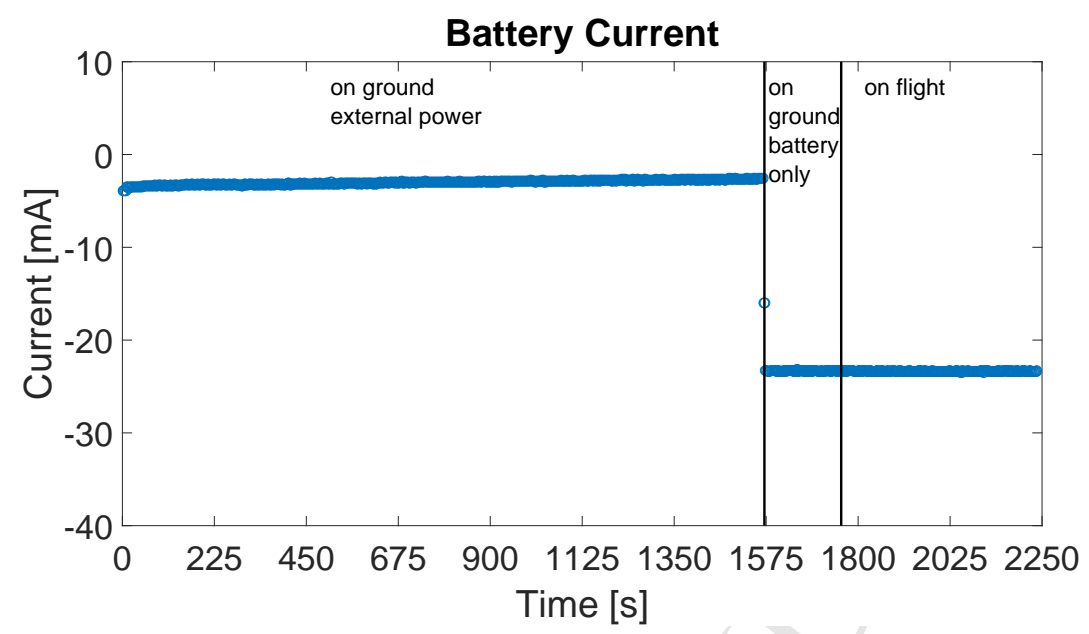

Figure 21: Battery current

flight. The battery monitoring chip is provided with an instantaneous current integrator, which allows the EPS to obtain battery state of charge information. This data is extremely valuable to the FloripaSat in order to schedule its tasks. Figure 22 shows a slightly decrease on the battery remained electric charge before the flight. This occurs, as explained above, due to the higher consuming current than the charging current. After the battery charging interruption, one may note that the battery discharging rate has increased. Also, this figure shows that during the flight, the battery discharging rate was the same than before the flight, since the subsystems power consumption has remained the same.

Figure 23 shows the battery monitoring chip internal temperature. A considerable temperature increase may be noted in this plot. Two are the main reasons for this effect: the first one is that the thermal experiment dissipates heat to the instrument aluminum casing (pre-heating operation mode), which leads to an overall temperature increasing inside the electronic compartment; the second reason is the chip internal self heating due to its operation. One should note that the heat dissipation to the instrument aluminum casing, due to the thermal experiment, may affect the thermal environment for the nanosatellite 


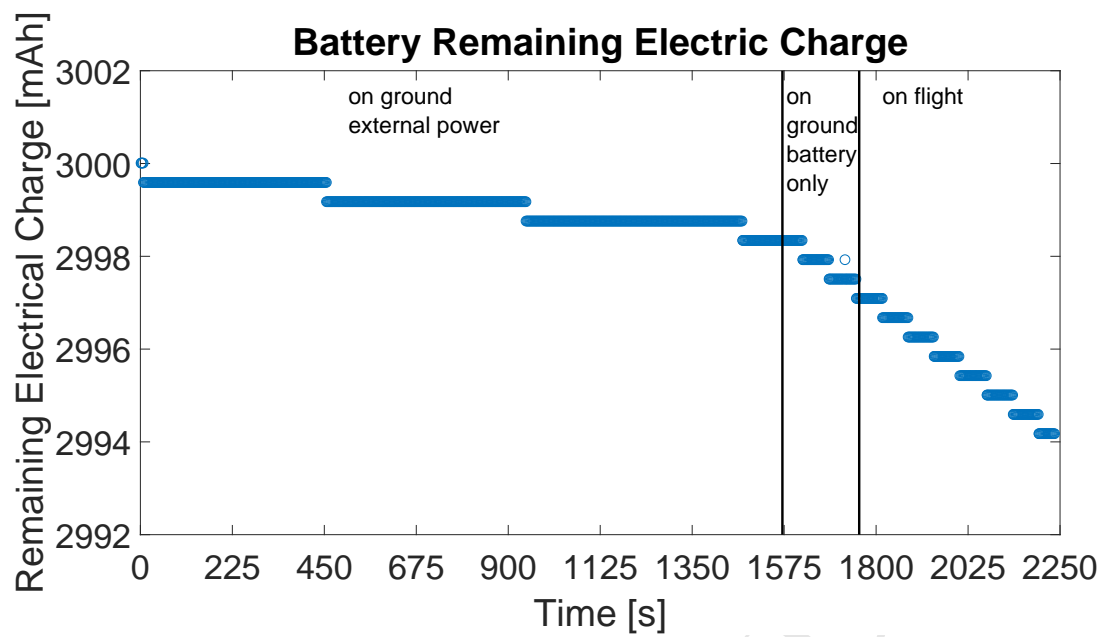

Figure 22: Battery remaining electric charge

subsystems tests. However, real environments, which would include transient boundary conditions, are very difficult and expensive to reproduce in the laboratory. Actually, the relevant observation here is that the temperature increasing rate was higher after the launch. Again, two reasons may have contributed to this: the first one is the rocket casing heating, due to the the air friction, which affects the whole experiment temperature behavior; the second reason is that the thermal experiment dissipated power was higher during the flight (heating up mode).

Figure 24 shows the OBDH microcontroller internal temperature. The temperature curve has a similar behavior than the one acquired by the EPS. This result confirms the consistence on the results, since two different sensors, in two different PCBs have registered similar results.

The temperature increase rate observed in Figures 23 and 24 is significantly higher than the ones applied on qualification tests (for instance, in thermal cycling test). The combination of harsh conditions verified along the rocket flight stage (high vibration, acceleration, mechanical shock) associated with this high increasing rate of temperature may cause satellite failures noted only 


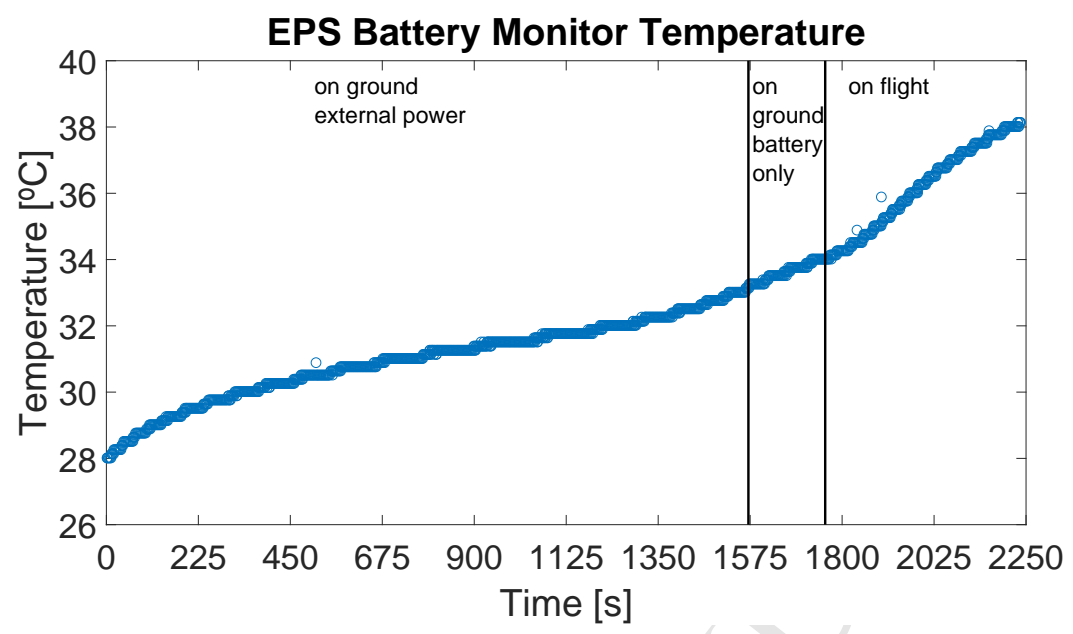

Figure 23: Battery monitoring chip internal temperature

when the spacecraft is in orbit. Therefore, the failures causes (or the failures themselves) may not be identifiable and recorded without the proposed test procedure or similar. Although the thermal environment inside the rocket is not representative when compared with the in-orbit condition, it may be considered a solution to record the nanosatellites' systems behavior during the rocket flight, as a matter of analysis, once all satellite shall face this mission stage.

Figure 25 shows the acceleration measurements performed by the $\mathrm{OBDH}$ IMU (MPU6050). From the plot, one may note only the g acceleration on z-axis before the launching, since the rocket z-axis was perpendicular to the Earth's surface at the launching platform. Also, as expected, there was no acceleration on axis $\mathrm{x}$ and $\mathrm{y}$ before the launching. Figure 25 shows the acceleration at the launching moment. Right after the lift-off, acceleration on $\mathrm{z}$ axis shows a peak of approximately $10 \mathrm{~g}$ and kept varying due to the ascendant movement of the rocket. As the rocket trajectory changes, $\mathrm{x}$ and $\mathrm{y}$ acceleration components were also increasing. Nearly at $1800 \mathrm{~s}$ the first stage separation occurred, causing high amplitudes on all axes. Due to a rocket belt separation fail, the acceleration level were higher than the expected and the rocket payload did not reach the correct 


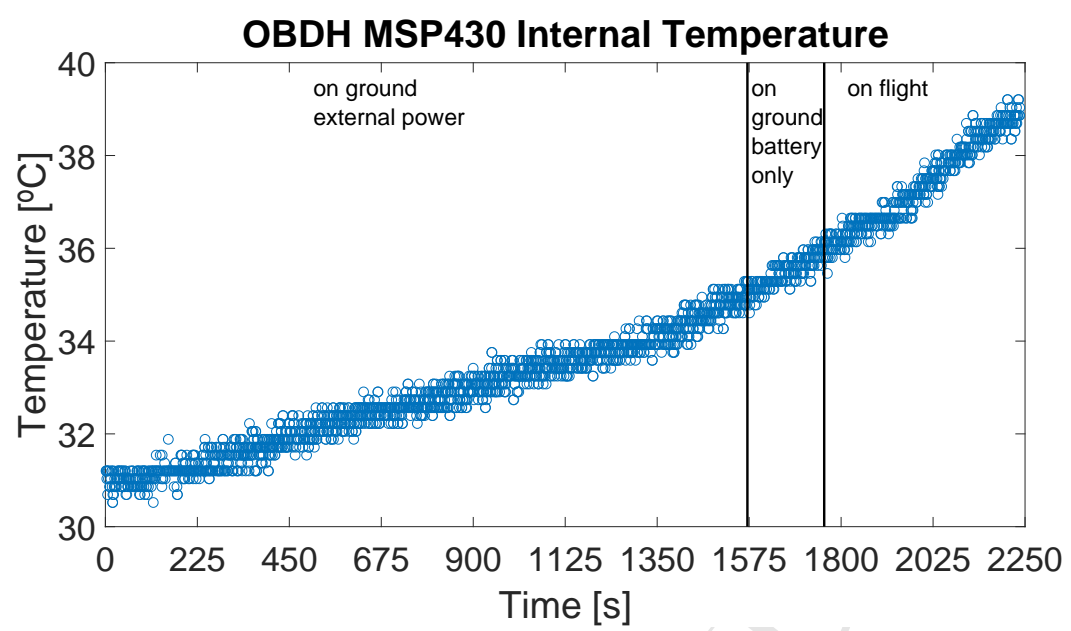

Figure 24: OBDH microcontroller internal temperature

velocity to obtain microgravity conditions. After $1830 \mathrm{~s}$ the rocket appears to be reached low gravity environment, but due to the reduced time, it may be interpreted as a free fall before the parachute opening. After $2050 \mathrm{~s}, \mathrm{z}$ axis component has returned to the same level before launching, and the other two axis acceleration returned to zero (rocket on vertical position).

Figure 26 shows the rotation measurements performed by the OBDH IMU. Before the launching, as the rocket was attached to the launching platform, no rotation was measured on any axis. After launching, fast rotation around $\mathrm{z}$ axis was recorded, due to the ascendant spinning movement of the rocket. The plot shows a saturation on the $\mathrm{z}$ axis measurement, which could be avoided using a dynamic IMU range reconfiguration. However, this rotation rate is not expected to occur when the satellite is in orbit. Therefore, no additional IMU reconfiguration software routine has been added for the sounding rocket mission. At $1800 \mathrm{~s}$, rocket attitude control was activated but due to the belt separation problem it failed. At $1900 \mathrm{~s}$ parachute was opened. Figure 26 one may also estimate the landing time (close to 2150 second in the plot), since no rotation is verified in all axes. 


\section{Acceleration}

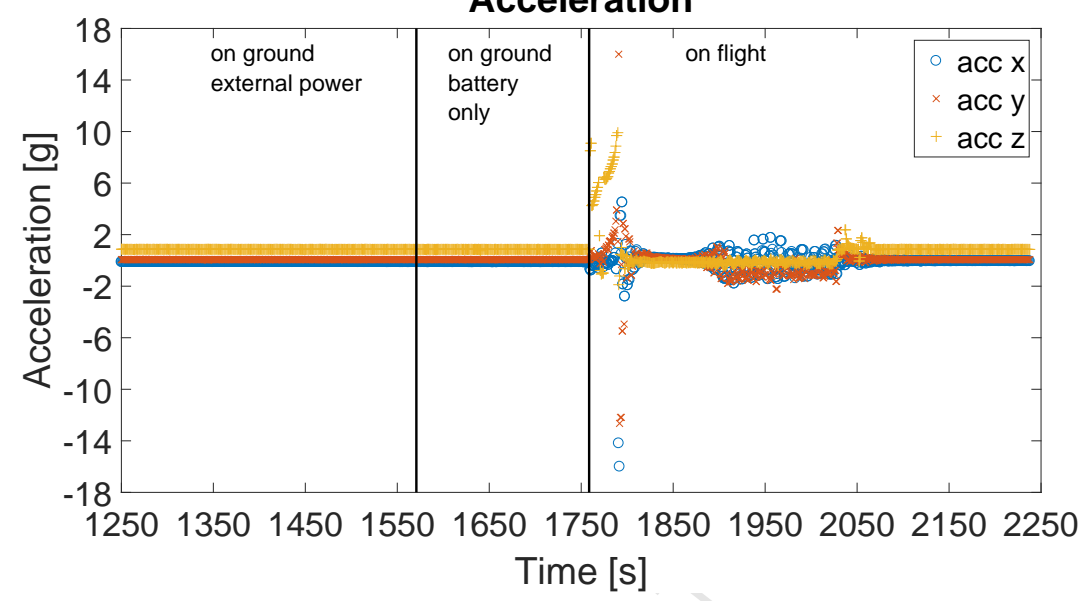

Figure 25: OBDH acceleration measurements

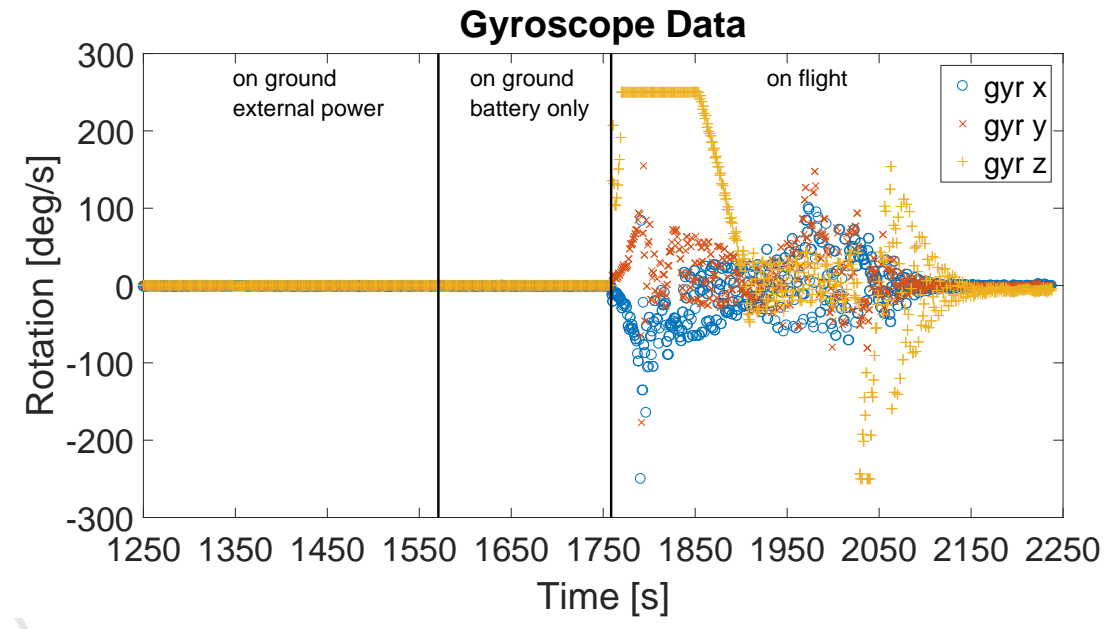

Figure 26: OBDH gyroscope measurements 
Unfortunately, there was no communication between the transceiver (CC1125) and the beacon transmitter (CC1175). Further analyses allowed to detect an intermittent communication fail between the radios, which was caused by a design mistake on the transceiver circuit. For the transceiver to work properly with the frequency used on this project $(437.5 \mathrm{MHz})$, the crystal should have frequencies higher than $40 \mathrm{MHz}$. The lack on attending this requirement has caused a frequency shifting on the transceiver. Although the beacon radio probably transmitted correctly (verified by operational tests before and after the flight), the transceiver did not receive the data. This hardware bug has already been corrected and tests with the new hardware are being carried out at the laboratory. Fortunately, most FloripaSat data was sent to the MPP through the OBDH communication (via UART), which allowed to test all other subsystems' functions.

Finally, a telemetry data loss shall be performed in order calculate the percentage of missed frames along the mission. This is an important parameter to evaluate the verification layers proposed both in the embedded software as well as in the ground station software. Since the rocket payload has been recovered, it was possible to compare the expected number of frames received by the ground station with the number of frames written in the OBDH internal memory. After computing both internal memory saved and telemetry received number of frames the conclusion was that $95.44 \%$ of the frames have been successfully received by the ground station.

Figure 27present a comparison between data received on the ground station and data saved in the OBDH internal memory along the flight for the OBDH microcontroller internal temperature measurement. One may note that there are slightly more points from the internal memory data than from the telemetry data, which represent the $4.56 \%$ on data losses. 


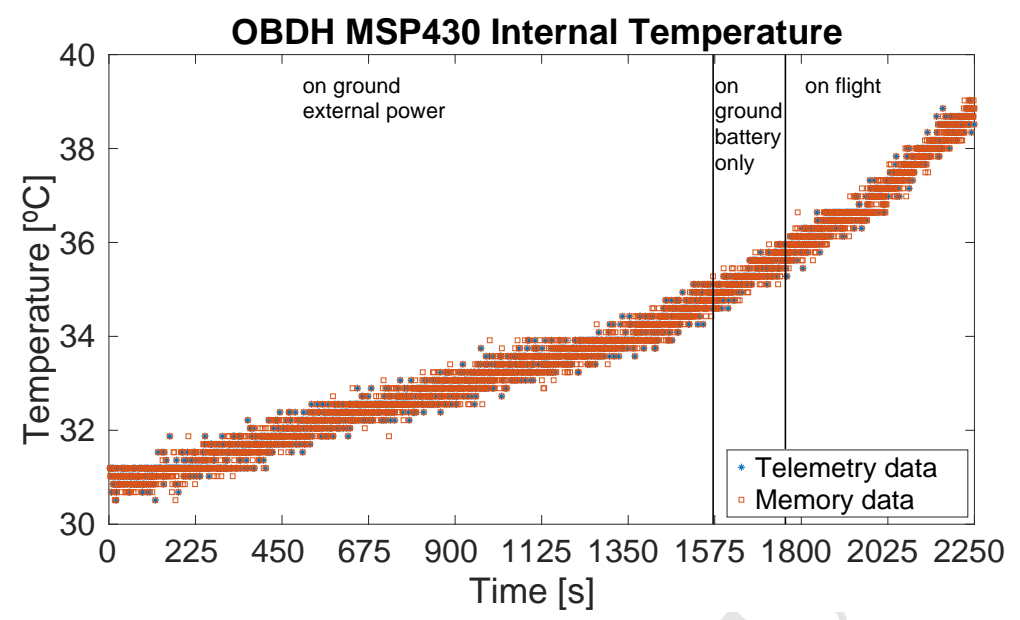

Figure 27: Telemetry - internal memory comparison: OBDH microcontroller internal temperature

\section{Conclusion}

This paper has presented a qualification test procedure for nanosatellites which allows analyzing the subsystems behavior under severe flight conditions. The idea behind the proposed test procedure, is to fly the engineering model of a nanosatellite in sounding rockets missions, before designing the satellite flight model. The work has described the hardware and software needs to build an interface between the rocket and the nanosatellite subsystems. The test interface (Multi-Mission Platform) allows to keep the tests as close as possible to the actual conditions that the satellite shall be submitted in the real mission. A case study has been presented, testing the FloripaSat engineering model subsystems, for which the sounding rocket flight results have guided to important modifications in the satellite flight model.

Besides the technical improvements obtained from the flight results analysis, the sounding rocket mission added operational and schedule constraints that have motivated the FloripaSat team. The mission requirements imposed by the DCTA have guided the team through procedures and methodologies that must be followed in space application projects. The experience obtained 
with the sounding rocket flight will certainly contribute to FloripaSat-I mission campaign, improving the team ability to manage the project schedule and to accomplish space mission requirements.

\section{References}

[1] D. Del Corso, C. Passerone, L. Reyneri, C. Sansoe, S. Speretta, M. Tranchero, Design of a university nano-satellite: the picpot case, Aerospace and Electronic Systems, IEEE Transactions on 47 (3) (2011) $1985-2007$.

口 [2] A. Poghosyan, A. Golkar, Cubesat evolution: Analyzing cubesat capabilities for conducting science missions, Progress in Aerospace Sciences 88

口 (2017) 59 - 83. doi:http://dx.doi.org/10.1016/j.paerosci.2016. 11.002 URL http://www.sciencedirect.com/science/article/pii/ S0376042116300951

[3] J. Bouwmeester, J. Guo, Survey of worldwide pico- and nanosatellite missions, distributions and subsystem technology, Acta Astronautica 67 (78) (2010) 854 - 862. doi:http://dx.doi.org/10.1016/j.actaastro.2010. 06.004

[4] S. Corpino, F. Stesina, Verification of a cubesat via hardware-in-the-loop simulation, IEEE Transactions on Aerospace and Electronic Systems 50 (4) (2014) 2807-2818. doi:10.1109/TAES.2014.130370

[5] X. Cao, Flexible platform based micro-satellite design method, Aerospace Science and Technology 53 (2016) 162 - 168. doi:http: //dx.doi.org/10.1016/j.ast.2016.03.012.

q [6] K. B. Gamble, E. G. Lightsey, Cubesat mission design software tool for risk estimating relationships, Acta Astronautica 102 (2014) 226 - 240. 
doi:http://dx.doi.org/10.1016/j.actaastro.2014.06.019.

URL http://www.sciencedirect.com/science/article/pii/ S0094576514002185

[7] Y. Miyazaki, M. Yamazaki, A practical education of space engineering by using cansat and pico-satellite - fruitful collaboration with unisec for success of student satellite program -, in: 2013 6th International Conference on Recent Advances in Space Technologies (RAST), 2013, pp. 1081-1086. doi : 10.1109/RAST.2013.6581163.

[8] S. Matunaga, K. Yoshihara, Y. Sugiura, M. Sekiguchi, H. Sawada, S. Tsurumi, K. Nakaya, M. Mori, K. Ui, N. Maeda, O. Mori, Titech micro-satellite model: Cansat for sub-orbital flight, in: 2000 IEEE Aerospace Conference. Proceedings (Cat. No.00TH8484), Vol. 7, 2000, pp. 135-142 vol.7. doi:10.1109/AER0.2000.879283.

q [9] K. V. Paiva, M. B. H. Mantelli, L. K. Slongo, Experimental testing a of mini heat pipes under microgravity conditions aboard a suborbital rocket, Aerospace Science and Technology 45 (2015) 367 - 375. doi:http://dx.doi.org/10.1016/j.ast.2015.06.004. URL //www.sciencedirect.com/science/article/pii/ S1270963815001819

[10] K. V. Paiva, M. B. H. Mantelli, L. K. Slongo, R. Gohr, V. B. Nicolau, Experimental tests of wire mini heat pipe under microgravity conditions aboard suborbital rockets, in: Proceedings of 9th International Heat Pipe Symposium, 2008.

[11] K. V. Paiva, M. B. H. Mantelli, G. V. Nuernberg, L. K. Slongo, F. Reis, S. J. Burg, V. B. Nicolau, Experimental tests of mini, pulsating and heat spreader heat pipes under microgravity conditions aboard suborbital rockets, in: Proceedings of 15th International Heat Pipe Conference, 2010.

[12] R. Varatharajoo, S. Fasoulas, Methodology for the developa ment of combined energy and attitude control systems for satel- 
lites, Aerospace Science and Technology 6 (4) (2002) 303 - 311. doi:http://dx.doi.org/10.1016/S1270-9638(02)01157-4.

[13] A. Addaim, A. Kherras, E. Zantou, Dsp implementation of integrated

a store-and-forward aprs payload and obdh subsystems for low-cost small satellite, Aerospace Science and Technology 12 (4) (2008) 308 - 317.

825 doi:http://dx.doi.org/10.1016/j.ast.2007.08.002. URL http://www.sciencedirect.com/science/article/pii/ S1270963807001034

[14] The CubeSat Program, Cubesat design specification rev. 13, Tech. rep., Cal Poly SLO (02 2014).

${ }_{830}$ [15] H. Chaoui, H. Gualous, Adaptive state of charge estimation of lithium-ion batteries with parameter and thermal uncertainties, IEEE Transactions on

【 Control Systems Technology 25 (2) (2017) 752-759. doi:10.1109/TCST. 2016.2572362

\section{Acknowledgement}

The authors acknowledge the Coordination for the Improvement of Higher Education Personnel (CAPES), which partially supported this research through the PhD grant number 3447735; the National Council for Scientific and Technological Development (CNPq), which partially supported this work under the project number 402184/2013-0, through the PhD grant number 141696/20176, and the postdoctoral grant number 150012/2018-7; and the Brazilian Space Agency (AEB), for having partially supported this work under Uniespaço Program, Opportunity Announce AO/2013 and Microgravidade Program, $4^{\text {th }}$ Opportunity Announce. 


\section{Authors' Biography}

Sara Vega Martínez is a Ph.D. student in Electrical Engineering at Federal University of Santa Catarina (UFSC), Brazil. She received her bachelor's and master's degrees in Electrical Engineering from University of Las Palmas 
de Gran Canarias (ULPGC), Spain, in 2016. Her research areas include embed-

ded systems hardware and software design, solar energy harvesting systems and nanosatellite electrical power systems.

Bruno Vale Barbosa Eiterer is a bachelor's student in Electrical Engineering at Federal University of Santa Catarina (UFSC), Brazil. His research areas include embedded systems software design, solar energy harvesting systems and nanosatellite electrical

power systems.

Túlio Gomez Pereira is a bachelor's student in Electrical Engineering at Federal University of Santa Catarina (UFSC). His research areas include embedded systems hardware design and test, solar energy harvesting systems and nanosatellite electrical power systems.

Mario Baldini Neto received his bachelor's degree in Computer Science and his master's degree in Electrical Engineering both at Federal University of Santa Catarina (UFSC), Brazil. His research areas include embedded operating systems focusing on nanosatellite on-board computers.

Matheus dos Santos Frata is a bachelor's student in Electronic Engineering at Federal University of Santa Catarina (UFSC). His research areas include embedded systems software design and test, and aerospace systems integration.

Henrique Daniel Hamisch is a bachelor's student in Control Engineering and Automation at Federal University of Santa Catarina (UFSC). His research areas include control and attitude systems design as well as embedded systems software

development.

André Martins Pio de Mattos is a bachelor's student in Electronic Engineering at Federal University of Santa Catarina (UFSC). His research areas include embedded systems software design, aerospace systems integration and test. 
Juan Pablo Florez Mera received his bachelor's degree in Mechanical Engineering from the Universidad Francisco de Paula Santander-UFPS, Colombia, in 2004, master's and Ph.D. degrees in Mechanical Engineering from the Federal University of Santa Catarina (UFSC), Brazil, in 2011 and 2016, respectively. His pipes.

Luigi Dilillo received his master's degree in Electronic Engineering from the Politecnico di Torino, Italy, in 2001, with specialization in Microelectron935 main research areas of interest is thermal control, working mainly in the following subjects: heat pipes, sintered porous media, LHP circuit heat pipes, and heat transfer mathematical modeling.

Kleber Vieira de Paiva is currently a professor at the Federal University of Santa Catarina (UFSC), Brazil. He is the header of the research group Thermal Fluid Flow Group - UFSC. He received his Ph.D. in Mechanical Engineering and Thermal Sciences from UFSC in 2011 and his master's degree in the same area and institution, in 2007. He has experience in the development of compact heat exchangers for thermal control of electronic components for space applications: mini heat pipes and thermosyphons.

Marcia Barbosa Henriques Mantelli received her bachelor's degree in Mechanical Engineering from the State University of Campinas, Brazil, in 1982, her master's degree in Space Engineering and Technology from the National Institute of Space Research, Brazil, in 1985 and her Ph.D. degree in Mechanical Engineering from the University of Waterloo, Canada, in 1995. She is currently professor at the Federal University of Santa Catarina (UFSC), Brazil and the header of the Heat Pipes Laboratory - LEPTEN/UFSC. She has experience in Mechanical Engineering, with emphasis on thermal engineering, working mainly on the following topics: satellites' thermal control, thermosyphons and heat

ics. He received his Ph.D. degree in Information, Structures and Systems 
from the University of Montpellier, France, in 2005. He did a post-doc at the University of Southampton, United Kingdom, from 2005 to 2007. He is currently a researcher for CNRS (1st class) at LIRMM University of Montpellier. His main research areas includes: memory test and reliability; power aware test; radiation impact on electronics; radiation monitoring; space and radiation hardened systems design; electronics working in harsh environment.

Eduardo Augusto Bezerra is a Researcher and Lecturer of Computer Engineering at Federal University of Santa Catarina (UFSC), where he is with 945 the Department of Electrical and Electronic Engineering since 2010. He received his Ph.D. in Computer Engineering from the University of Sussex (Space Science Centre), England, UK, in 2002. His research interests are in the areas of embedded systems, space applications, computer architecture, reconfigurable systems (FPGAs), software \& hardware testing, fault tolerance and microprocessor applications. 
Bruno Vale Barbosa Eiterer

Tulio Gomes Pereira

Mario Baldini Neto, M.Eng.

Matheus dos Santos Frata

Henrique Daniel Hamisch

André Martins Pio de Mattos

Prof. Eduardo Augusto Bezerra, PhD

Federal University of Santa Catarina

Federal University of Santa Catarina

Technological Center (CTC)

Department of Electrical and Electronic Engineering Technological Center (CTC)

Embedded Systems Group (GSE)

Florianópolis, Zip Code 88040-900, Brazil

E-Mail: slongo@eel.ufsc.br; jgreis@lisha.ufsc.br; sara.vega@posgrad.ufsc.br; bruno.eiterer@grad.ufsc.br; tulio.gomes. pereira@grad.ufsc.br;

mario.baldini@posgrad.ufsc.br; m.frata@grad.ufsc.br;

henrique.hamisch@grad.ufsc.br;

andre.martins.mattos@grad.ufsc.br

Eduardo.Bezerra@ufsc.br;

Daniel Gaiki, B.Eng.

Luigi Dilillo, $\mathrm{PhD}$

Politecnico di Torino

Dipartimento di Elettronica e Telecomunicazioni

Turin, Corso Castelfidardo, 39, Italy

E-Mail: daniel.gaiki@studenti.polito.it

Department of Mechanical Engineering

Heat Pipes Laboratory

Florianópolis, Zip Code 88040-900, Brazil

E-Mail: pedro@labtucal.ufsc.br; jpablo@labtucal.ufsc.br; marcia.mantelli@ufsc.br

Prof. Kleber Vieira de Paiva, Dr.Eng.

Federal University of Santa Catarina

Technological Center of Joinville

Department of Mobility Engineering

Aerospace Engineering

Thermal Fluid Flow Group

Joinville, Zip Code 89218-035, Brazil

E-Mail: kleber.paiva@ufsc.br

November 20, 2018

Acta Astronautica, Editor-in-Chief

Dear Prof. Rock Jeng-Shing Chern, PhD,

We would like to submit the attached manuscript, "Pre-flight qualification test procedure for nanosatellites using sounding rockets", for consideration for possible publication in Acta Astronautica.

The highlights of the paper are the following:

- A detailed description of a qualification test procedure for nanosatellites.

- Embedded system to interface the nanosatellite subsystems and the rocket electronics.

- Design description, test and on-flight validation of a Multi-Mission Platform.

- Ground station data processing software.

- Nanosatellite subsystems test results from a sounding rocket flight.

Sincerely, 